\title{
Étude en laboratoire des propriétés mécaniques de l'argile molle de Guiche (vallée de l'Adour)
}

M. KHEMISSA,

J.-P. MAGNAN,

H. JOSSEAUME

Laboratoire Central des Ponts et Chaussées 58, boulevard Lefebvre,

75732 Paris Cedex 15
[O Le comportement mécanique de l'argile molle de Guiche a été étudié au moyen d'essais œdométriques conventionnels à chargement par paliers et d'essais triaxiaux de divers types à l'appareil triaxial classique. L'étude visait à caractériser les relations entre les contraintes effectives, les déformations et le temps et leur effet sur le comportement à l'état limite de l'argile. Les essais ont été réalisés sur des carottes prélevées entre 10 et $15 \mathrm{~m}$ de profondeur au moyen de carottiers à piston stationnaire de 100 et $200 \mathrm{~mm}$ de diamètre. Malgré des incertitudes liées au remaniement possible des éprouvettes testées, l'étude a permis de déterminer une courbe d'état limite de forme elliptique, une loi d'écoulement non associée, une loi de fluage semilogarithmique et un comportement surconsolidé de type anisotrope. Ces résultats confirment les conclusions des études effectuées sur d'autres argiles naturelles comparables.

Mots clés : argile, triaxial, œdomètre, état limite, anisotropie, fluage.

\section{Laboratory study of the mechanical properties of Guiche soft clay (Adour valley)}

Conventional oedometric multi-stage loading and various types of classical triaxial tests were performed on Guiche soft clay for to study its mechanical properties. This research was aimed at characterising the stress-strain-time relationship and its effect on yielding of the clay. The specimens used for this study were taken at a depth of 10 to $15 \mathrm{~m}$, using 100 and $200 \mathrm{~mm}$ diameter samplers. Despite the uncertainties associated with the potential for destructuration of the test specimens, the study allowed to characterize the behaviour of this soil by exhibiting an elliptical limit state curve, a non associated flow rule, a semi-logarithmic creep law and an anisotropic overconsolidated response. These results confirm the conclusions derived from other studies on similar natural clays.

Key words : clay, triaxial test, oedometer test, limit state, anisotropy, creep. 


\section{1}

\section{Introduction}

Depuis une trentaine d'années, les Laboratoires des ponts et chaussées (France) étudient les propriétés mécaniques des sols naturels, notamment des argiles, afin de mieux comprendre leur comportement observé sous les ouvrages de génie civil et d'alimenter les modèles de calcul en données expérimentales fiables et représentatives. Les premières études systématiques en laboratoire ont été consacrées à l'argile molle de Lanester (Josseaume et Meimon, 1976; Josseaume et al., 1977). Elles ont été suivies, pendant une dizaine d'années, d'études sur l'argile molle du site expérimental de remblais sur sols mous de Cubzac-les-Ponts (vallée de la Dordogne), dont on a défini successivement le comportement non drainé (Vogien, 1975), la courbe d'état limite (Magnan et al., 1982), le comportement surconsolidé anisotrope (Piyal et Magnan, 1984; Magnan et Piyal, 1985) et certains aspects viscoplastiques de la loi de comportement (Kenana et Félix, 1989). L'étude de l'argile de Guiche (vallée de l'Adour), dont les résultats sont décrits dans le présent article, a été engagée en parallèle à la construction d'autres remblais expérimentaux à la fin des années 1980. Pendant cette période, d'autres études en laboratoire, suivant des méthodologies voisines, ont été réalisées sur deux argiles raides: l'argile des Flandres (Josseaume et al., 1991), dont les propriétés mécaniques sont proches de celles de l'argile de Londres (Graham et al., 1983), et l'argile verte de Romainville (Josseaume et Azizi, 1991), ainsi que sur des marnes de différentes origines (Laboratoire régional des ponts et chaussées d'Aix-en-Provence) et sur des matériaux de remblais compactés (Laboratoire régional des ponts et chaussées de Toulouse).

La démarche adoptée pour ces études expérimentales reflète d'une certaine façon la structure des lois de comportement qui sont utilisées en parallèle pour la modélisation du comportement des massifs de sols :

- les premières études sur les argiles de Lanester et de Cubzac-les-Ponts ont été effectuées à une époque où l'on considérait comme acquis, après les travaux de Bjerrum (1967, 1973), que les massifs d'argile avaient d'abord un comportement non drainé pendant l'application de la charge, puis un comportement de consolidation une fois la charge stabilisée. Les études en contraintes totales avaient pour but unique de caractériser le comportement non drainé du sol pendant l'application du chargement, en tenant compte de la vitesse de chargement, et devaient alimenter des lois de comportement de type élastoplastique anisotrope ; - à la fin des années 1970, à la suite notamment des travaux réalisés en collaboration par l'université Laval de Québec et par les Laboratoires des ponts et chaussées en France (Leroueil et al., 1985), il était clairement préférable d'écrire les lois de comportement des argiles comme des autres sols saturés en termes de contraintes effectives et d'alimenter des lois de comportement dérivées des modèles de l'université de Cambridge et tenant compte en particulier des effets du temps. Les études expérimentales effectuées sur l'argile de Cubzac-les-Ponts ont d'abord repris les procédures utilisées à l'université Laval pour l'étude des argiles sensibles de la vallée du Saint-Laurent (Tavenas et Leroueil, 1979; Leroueil et al., 1983) pour la détermination de la courbe d'état limite, puis ont été complétées par des procédures d'étude de l'anisotropie des déformations. Le modèle de comportement sous-jacent à ces études est le modèle «Mélanie », utilisé notamment pour les études numériques par éléments finis au Laboratoire central des ponts et chaussées et que nous considérons comme équivalent aux modèles reliant les contraintes effectives, les déformations et les vitesses de déformation :

- quelques études expérimentales ont été exécutées avec une autre référence théorique qui est la viscoélasticité (et la viscoplasticité), sous la forme utilisée notamment dans l'ancienne Union soviétique (Félix, 1980), mais cette approche a été ultérieurement abandonnée.

L'étude expérimentale de l'argile de Guiche a comporté différents types d'essais triaxiaux et œdométriques. Elle avait pour objectifs :

- la détermination de la courbe d'état limite de l'argile; - la mesure de l'anisotropie de l'argile à l'état surconsolidé ;

- la caractérisation de la compressibilité et de la perméabilité de l'argile à l'cedomètre ;

- l'étude des effets du temps (fluage).

Après une présentation rapide de l'argile, cet article décrit le programme expérimental et les procédures d'essais, les résultats des essais et leur interprétation en termes de courbe d'état limite, d'anisotropie, de fluage, de compressibilité œdométrique et de perméabilité.

\section{2}

\section{L'argile de Guiche}

Les échantillons d'argile utilisés pour la présente étude ont été prélevés par le Laboratoire régional des ponts et chaussées de Bordeaux, dans le cadre de la reconnaissance géotechnique du site expérimental de Guiche, situé dans la basse vallée de l'Adour, où des remblais d'essai ont été édifiés à la fin des années 1980 (Queyroi et al., 1991). La basse vallée de l'Adour est assez bien connue au plan géotechnique, suite aux nombreuses reconnaissances effectuées pour le tracé de l'autoroute A64 entre Peyrehorade et Bayonne (Magnan et al., 1987). On y trouve suivant les endroits de 20 à $40 \mathrm{~m}$ de dépôts récents d'argile molle, de tourbe et de sable, reposant sur des graviers et des blocs de rochers issus de l'érosion des Pyrénées voisines. Dans la zone de Guiche, la coupe géotechnique type comporte à partir de la surface du terrain naturel (Fig. 1) : - une croûte d'argile limoneuse surconsolidée d'environ 2 m d'épaisseur ;

- une couche d'argile limoneuse faiblement organique de 2 à $3 \mathrm{~m}$ d'épaisseur ;

- une couche de tourbe fibreuse légèrement surconsolidée d'épaisseur variable entre 1 et $1,5 \mathrm{~m}$;

- une couche d'argile limoneuse faiblement organique de 9 à $14 \mathrm{~m}$ d'épaisseur :

- une couche de grave sableuse, qui sert de substratum pour les études effectuées sur le site.

Les caractéristiques géotechniques moyennes de ces couches sont données dans le tableau I.

La couche d'argile limoneuse faiblement organique qui s'étend de 6 à $20 \mathrm{~m}$ de profondeur à l'emplacement des sondages présente des caractéristiques géotechniques 

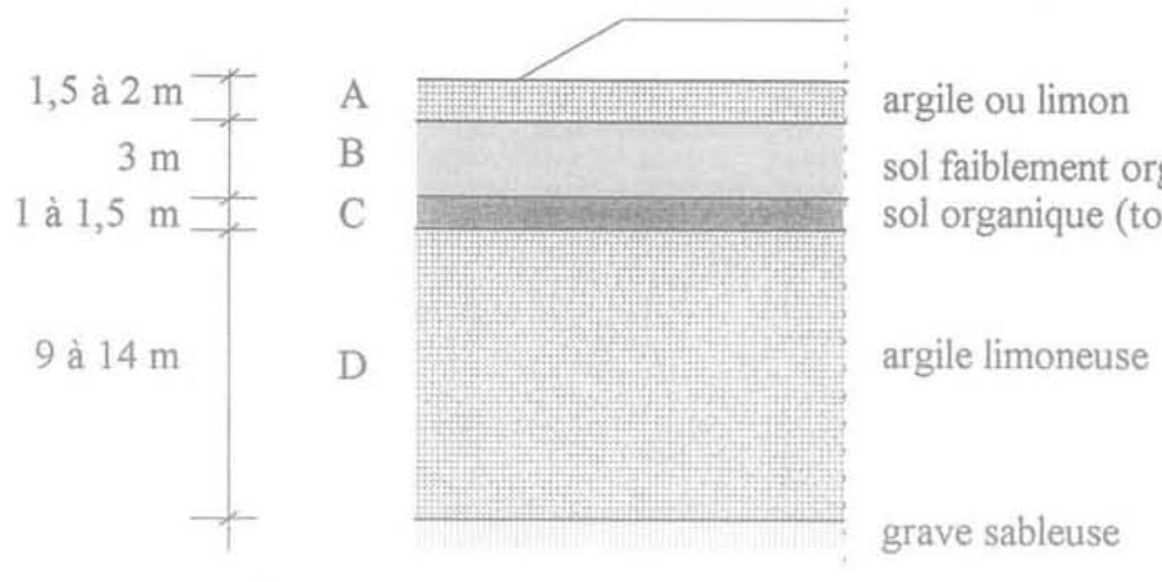

FiG.1 Profil géotechnique type des couches de sols sur le site expérimental de Guiche (vallée de l'Adour). Typical geotechnical profile of soil layers at the Guiche test site (Adour valley).

ThaLEAUt Valeurs moyennes des caractéristiques géotechniques des couches de sols sur le site expérimental de Guiche (vallée de l'Adour).

Mean values of the geotechnical characteristics of the soil layers at the Guiche test site (Adour valley).

\begin{tabular}{|c|c|c|c|c|c|c|c|c|}
\hline Couche & $\begin{array}{l}\text { Épaisseur } \\
\text { (m) }\end{array}$ & $\begin{array}{l}\text { Classification LPC } \\
\text { (Magnan, 1980) }\end{array}$ & w & $\frac{\gamma}{\left(\mathrm{kN} / \mathrm{m}^{3}\right)}$ & $I_{p}$ & $C_{3 \omega 0}$ & $e_{a}$ & $(\mathrm{kPa})$ \\
\hline A & $1,5-2$ & At & 45 & 17,4 & 35 & - & 1,11 & 58 \\
\hline B & 3 & fo-At & 95 & 14,6 & 59 & 6,2 & 2,10 & 25 \\
\hline C & $1-1,5$ & to-f & 362 & 10,6 & - & 67 & 5,85 & - \\
\hline D & $9-14$ & fo-Lt & 55 & 16,3 & 49 & 4,3 & 1,61 & 26 \\
\hline
\end{tabular}

At: argile très plastique; fo-At: argile très plastique faiblement organique; to-f: tourbe fibreuse; fo-L: limon très plastique faiblement organique.

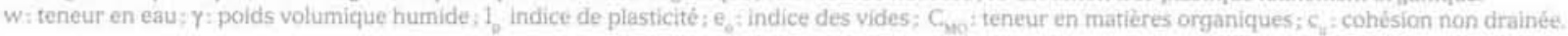

relativement homogènes. Selon la classification LPC (Magnan, 1980), il s'agit d'une argile molle, limoneuse, faiblement organique et très plastique (fo-Lt).

Les carottes d'argile utilisées pour la présente étude ont été prélevées dans trois sondages distants de quelques mètres, au moyen d'un carottier à piston stationnaire de $100 \mathrm{~mm}$ de diamètre de type LPC (Lemasson, 1973) pour le sondage F0 et au moyen d'un carottier à piston stationnaire et enveloppe rotative de $200 \mathrm{~mm}$ de diamètre, semblable à celui développé à l'université Laval (La Rochelle et al., 1981), pour les sondages F1 et F2. Ce dernier carottier fournit des carottes de $600 \mathrm{~mm}$ de hauteur, dans lesquelles il est possible de tailler jusqu'à six éprouvettes cedométriques ou huit éprouvettes triaxiales au même niveau.

\section{3}

\section{Programme expérimental et procédures d'essais}

Le programme expérimental a comporté 43 essais œdométriques et 50 essais triaxiaux, que l'on peut regrouper en neuf séries :

- une série (O1) de 35 essais de compressibilité œdométriques à chargement par poids et par paliers de 24 heures, selon les méthodes d'essai LPC (Magnan et al., 1985) : taux d'incrément de charge de 1,5 avant le coude de la courbe de compressibilité, puis de 2 ; boucle de déchargement et rechargement en deux étapes dès que l'on a mis en évidence le coude de la courbe de compressibilité ;

- une série (O2) de 8 essais de fluage à l'œedomètre, sur des éprouvettes soumises chacune à une charge unique maintenue pendant trois mois. Les charges appliquées couvrent la même gamme de contraintes que les essais de compressibilité œdométriques à chargement par poids :

- trois séries d'essais triaxiaux de compression non drainés, sur des éprouvettes consolidées sous contraintes isotropes (T3 : 21 essais CIU), sur des éprouvettes reconsolidées aux contraintes effectives en place (T4 : 2 essais CK U) et sur des éprouvettes reconsolidées aux contraintes effectives en place puis consolidées sous contraintes isotropes (T5 : 6 essais CK IU) ; - deux séries d’essais triaxiaux d'extension non drainés sur des éprouvettes reconsolidées aux contraintes effectives en place (T6 : 1 essai EK U) et sur des éprouvettes reconsolidées aux contraintes effectives en place puis consolidées sous des contraintes isotropes (T7:3 essais EK IU); - une série de 7 essais triaxiaux de consolidation anisotrope sur des éprouvettes soumises à des chargements par paliers de 24 heures, selon des chemins de contraintes à rapport des contraintes effectives radiale et axiale constant (T8:7 essais CAP) ;

- une série de 10 essais triaxiaux de compression drainés, sur des éprouvettes verticales découpées parallèlement à l'axe vertical de la couche d'argile (T9/1: 5 essais CID) et sur des éprouvettes horizontales découpées perpendiculairement à cet axe (T9/2: 5 essais CID), reconsolidées sous la pression moyenne effective des terres en place. 
taBleau II Origine des éprouvettes testées dans les différentes séries d'essais.

Origin of the specimens tested in the different test series.

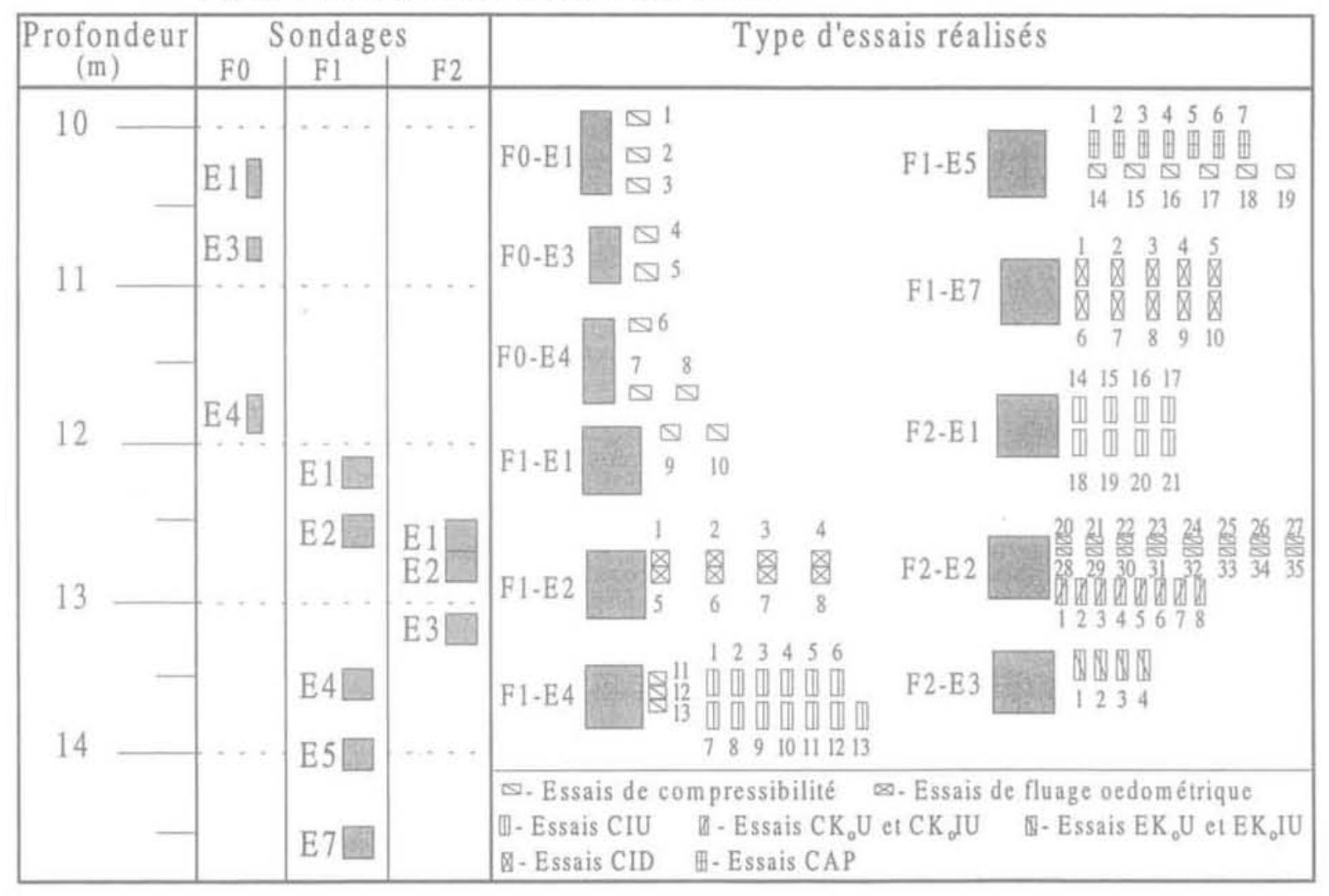

L'origine des éprouvettes testées (profondeur, sondage, type de carottier, carotte) est indiquée dans le tableau II, avec le type d'essai réalisé.

Neuf essais œdométriques et quatorze essais triaxiaux ont été écartés pour remaniement excessif des éprouvettes correspondantes. Les critères de remaniement adoptés sont au nombre de quatre (Khemissa, 1992; Khemissa et al., 1993) :

- le premier critère est défini pour les essais œedométriques et se traduit, pour une éprouvette remaniée, par des valeurs de la pression de préconsolidation assez faibles et inférieures aux valeurs de la pression effective des terres en place, c'est-à-dire par des rapports de surconsolidation $\mathrm{R}_{\mathrm{oc}}=\sigma_{\mathrm{p}}^{\prime} / \sigma_{\mathrm{yo}}^{\prime}$ inférieures à 1;

- le deuxième critère est défini à partir des résultats d'essais triaxiaux de compression et d'extension non drainés sur éprouvettes reconsolidées aux contraintes effectives en place : les valeurs de la déformation volumique en fin de consolidation y sont d'autant plus élevées que l'argile est plus remaniée;

- le troisième critère s'applique aussi aux résultats d'essais triaxiaux de compression et d'extension non drainés. Il affirme que la cohésion non drainée dans une éprouvette remaniée est plus faible que celle caractérisant une éprouvette intacte testée dans les mêmes conditions ;

- le quatrième critère est défini à partir des résultats d'essais triaxiaux de compression et d'extension non drainés et se traduit, pour une éprouvette remaniée, par des valeurs des modules de déformabilité non drainés plus faibles que celles obtenues pour une éprouvette intacte testée dans les mêmes conditions. De même, toutes choses égales par ailleurs, la valeur de la déformation axiale à la rupture est plus faible pour une éprouvette intacte que pour une éprouvette remaniée.
Les essais de compressibilité et de fluage œdométriques ont été exécutés dans des œdomètres à chargement par poids, de type Wykeham Farrance, sur des éprouvettes de $60 \mathrm{~mm}$ de diamètre et $20 \mathrm{~mm}$ d'épaisseur initiale. Les essais triaxiaux ont été exécutés au moyen de cellules triaxiales classiques, de type Wykeham Farrance, modifiées pour l'acquisition des mesures et le contrôle des chargements par ordinateur. Ces appareils sont rassemblés dans une salle climatisée à $20 \pm 1{ }^{\circ} \mathrm{C}$. Les procédures des essais cedométriques réalisés ont été rappelées brièvement ci-dessus. Les opérations effectuées successivement dans les différentes séries d'essais triaxiaux réalisés comportent: - une phase initiale commune à l'ensemble des essais triaxiaux qui comporte le montage à sec et la saturation des circuits de drainage, la mesure de la pression interstitielle initiale u et l'application d'une contre-pression isotrope $\mathrm{u}_{\mathrm{cp}}$;

- une phase de chargement par paliers de 24 heures à chemin de contraintes contrôlé ( $\sigma_{\mathrm{a}}$ et $\sigma$, variables) pour les essais CAP ;

- une phase de consolidation isotrope sous une contrainte $\sigma_{c}^{\prime}$ pour les essais CIU et CID ou de reconsolidation aux contraintes effectives en place $\left(\sigma^{\prime}{ }_{v o}, K_{0} \sigma_{v 0}^{\prime}\right)$ avec $\mathrm{K}_{0}=0,55$ pour les essais $\mathrm{CK}_{0} \mathrm{U}, \mathrm{CK}_{0} \mathrm{IU}, \mathrm{EK}_{\mathrm{o}} \mathrm{U}$ et EK IU. Après annulation du déviateur des contraintes à volume constant et à contrainte radiale constante et après application d'une pression cellulaire $\sigma_{\text {co }}^{\prime}$ telle que la pression interstitielle $\mathrm{u}=\mathrm{u}_{c p}$ la reconsolidation aux contraintes effectives en place des éprouvettes utilisées dans les essais CK IU et EK IU a été suivie d'une nouvelle consolidation isotrope sous une contrainte $\sigma_{c}^{\prime} \neq \sigma_{c o}^{\prime}$; - une phase de cisaillement drainé pour les essais CID ou non drainé en compression pour les essais CIU, $\mathrm{CK}_{0} \mathrm{U}$ et $\mathrm{CK}_{0} \mathrm{IU}$ et en extension pour les essais $\mathrm{EK}_{\mathrm{o}} \mathrm{U}$ et 
EK IU à vitesse de déformation constante et à contrainte radiale constante.

\section{4}

\section{Résultats des essais}

\section{1}

\section{Essais de compressibilité œdométriques}

La figure 2 présente les courbes de compressibilité de l'ensemble des essais œdométriques. Les indices des vides initiaux e varient de 1,56 à 1,95 et les courbes «e$\lg \sigma^{\prime}$ n donnent une impression de variabilité qui disparait lorsqu'on représente la déformation verticale relative $\varepsilon_{\text {out }}=\Delta \mathrm{e} /(1+\mathrm{e})$ en fonction du logarithme de la contrainte effective $\sigma$, comme on l'a fait sur la figure 2.

Les coudes correspondant à la pression de préconsolidation sont assez bien marqués sur la plupart des courbes de compressibilité. Chaque point de ces courbes correspond à la déformation verticale relative observée au bout de 24 heures sous la charge appliquée (contrainte effective finale).

La figure 3 présente les courbes de compressibilité (c e-lgo' $\sigma^{\prime}$, de perméabilité " e-lgk ») et de consolidation « $\Delta \mathrm{H}-\sqrt{t} »$ d'un des essais (essai $n^{\circ} 24$ ), avec les équations des courbes de compressibilité et de perméabilité et les valeurs des coefficients de perméabilité $k_{y}$ et de consolidation $\mathrm{c}_{\mathrm{v}}$ et du temps $\mathrm{t}_{90}$ (correspondant à $90 \%$ de consolidation primaire) déterminés par la méthode de Taylor sous les paliers de chargement correspondant aux contraintes supérieures à la pression de préconsolidation de l'argile.

\section{Essais de fluage œdométriques}

Les courbes de tassement en fonction du logarithme du temps des huit éprouvettes, qui ont été soumises pendant trois mois à huit charges constantes différentes, sont représentées sur la figure 4. Elles ont des allures classiques, avec une partie initiale de consolidation primaire suivie d'une partie linéaire correspondant à la consolidation secondaire caractérisée par le taux de fluage $\mathrm{C}_{\alpha}=$ $\left[\Delta \mathrm{H}_{/} / \mathrm{H}_{\mathrm{c}}\right] /[\Delta \mathrm{lgt}]$, où $\mathrm{H}_{\mathrm{o}}=20 \mathrm{~mm}$ désigne la hauteur initiale des éprouvettes. L'intersection de ces deux parties correspond au temps $t_{100}$ de consolidation primaire.

Les courbes de tassement présentent toutefois, après $20000 \mathrm{~min}$, un décrochement de forme comparable à celle de la consolidation primaire, dont la seule explication plausible est la panne du système de climatisation de la salle d'essai pendant la canicule de juin 1990 , où les températures diurnes dépassaient $40^{\circ} \mathrm{C}$. La courbe $7\left(\sigma^{\prime}=420 \mathrm{kPa}\right)$ présente aussi une forme atypique, avec une consolidation primaire anormalement longue. Ce phénomène peut être dû à un certain remaniement de l'éprouvette testée.

\section{3}

\section{Essais triaxiaux de compression et d'extension}

Les résultats de tous les essais triaxiaux de compression et d'extension drainés et non drainés et de consolidation anisotrope sont représentés sur les figures 5 à 9 .

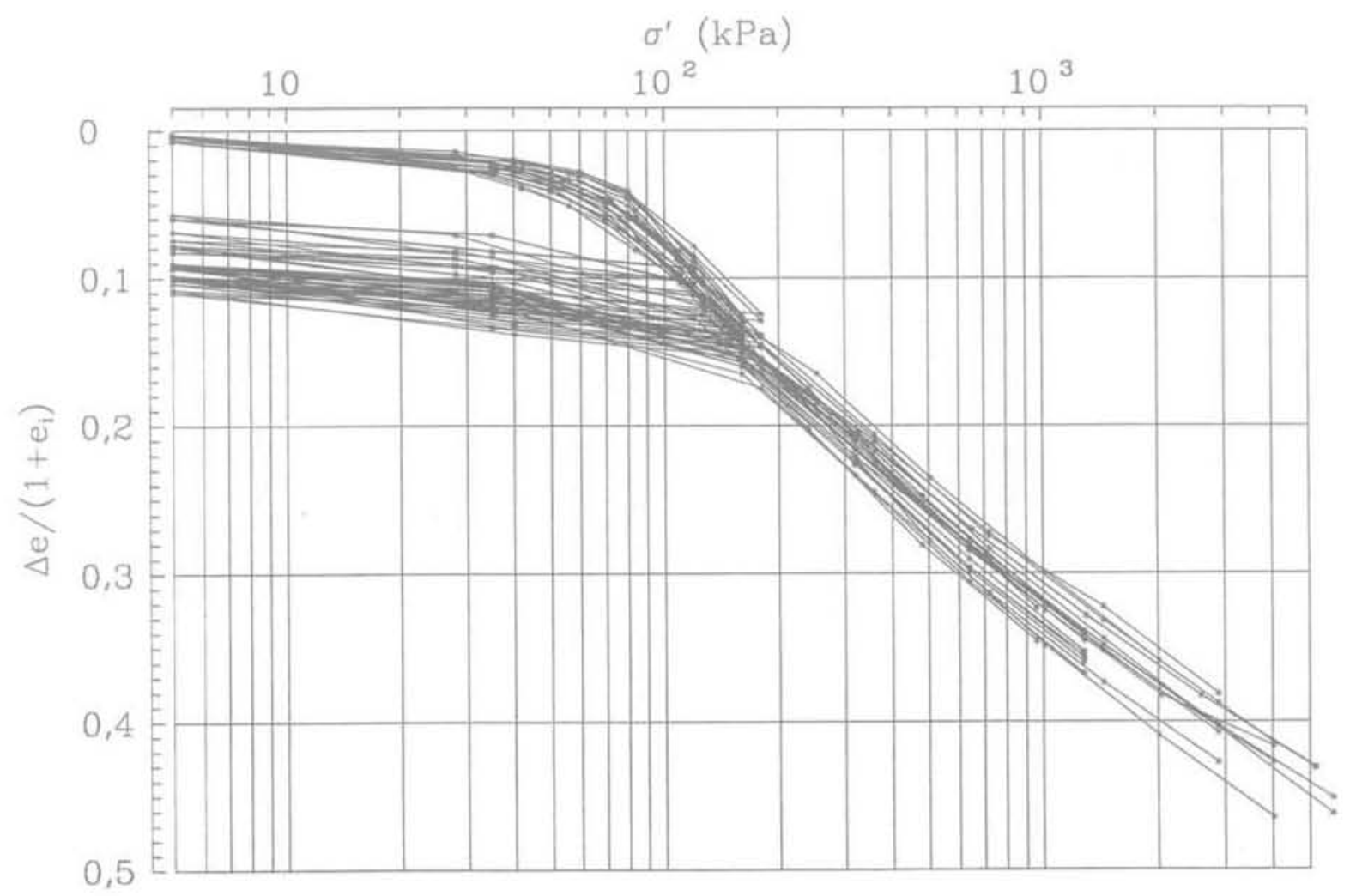

F1G. 2 Courbes de compressibilité des essais œdométriques (déformation relative à 24 heures en fonction de la pression appliquée). Compression curves derived from the oedometer tests (strains at 24 hours versus applied pressure). 

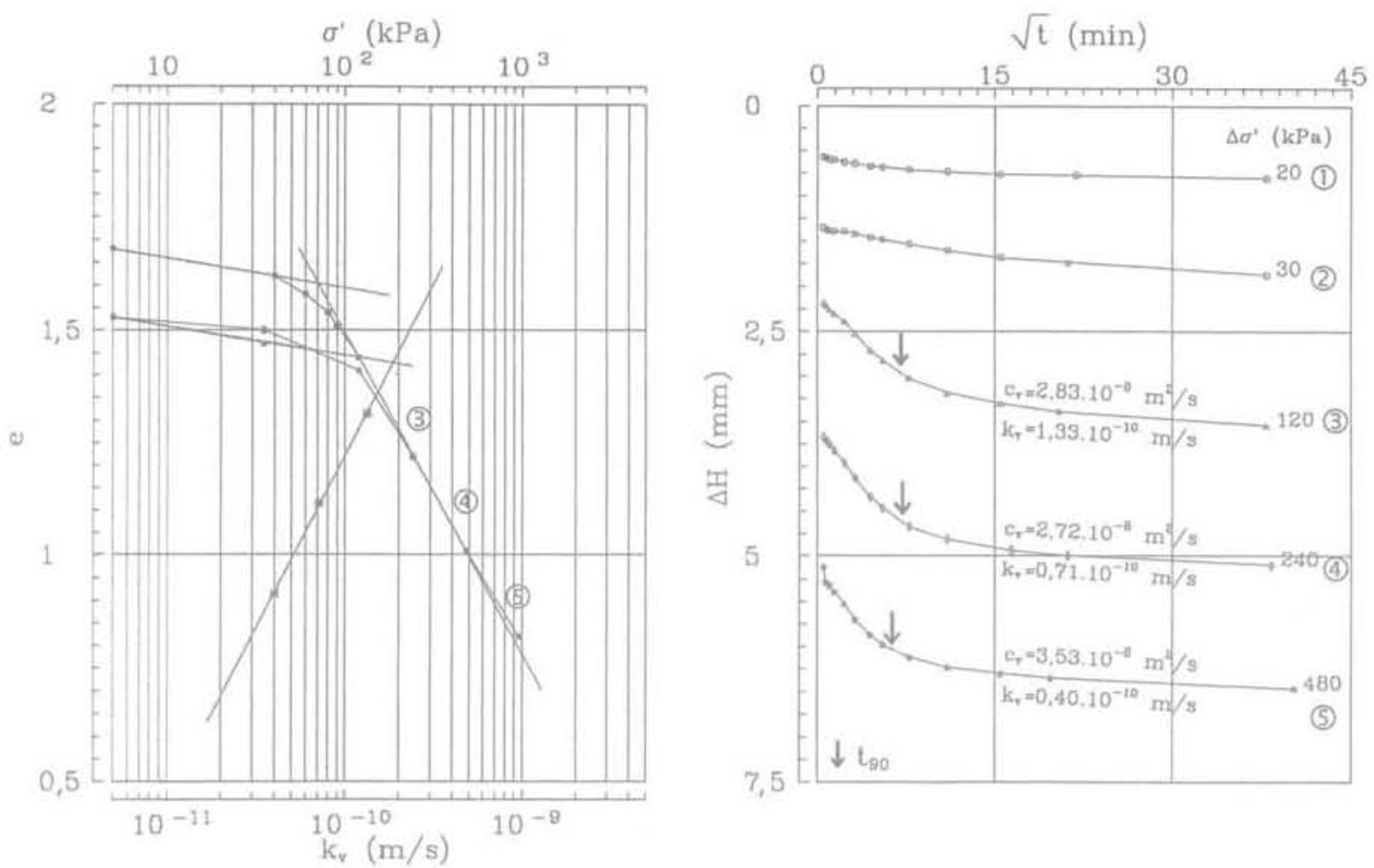

FiG.3 Courbes de compressibilité, de perméabilité et de consolidation sous les paliers de chargement successifs d'un essai œdométrique (essai n²4).

Compression, permeability and consolidation curves obtained from oedometer tests carried out under increased loads (test $\left.n^{\circ} 24\right)$.

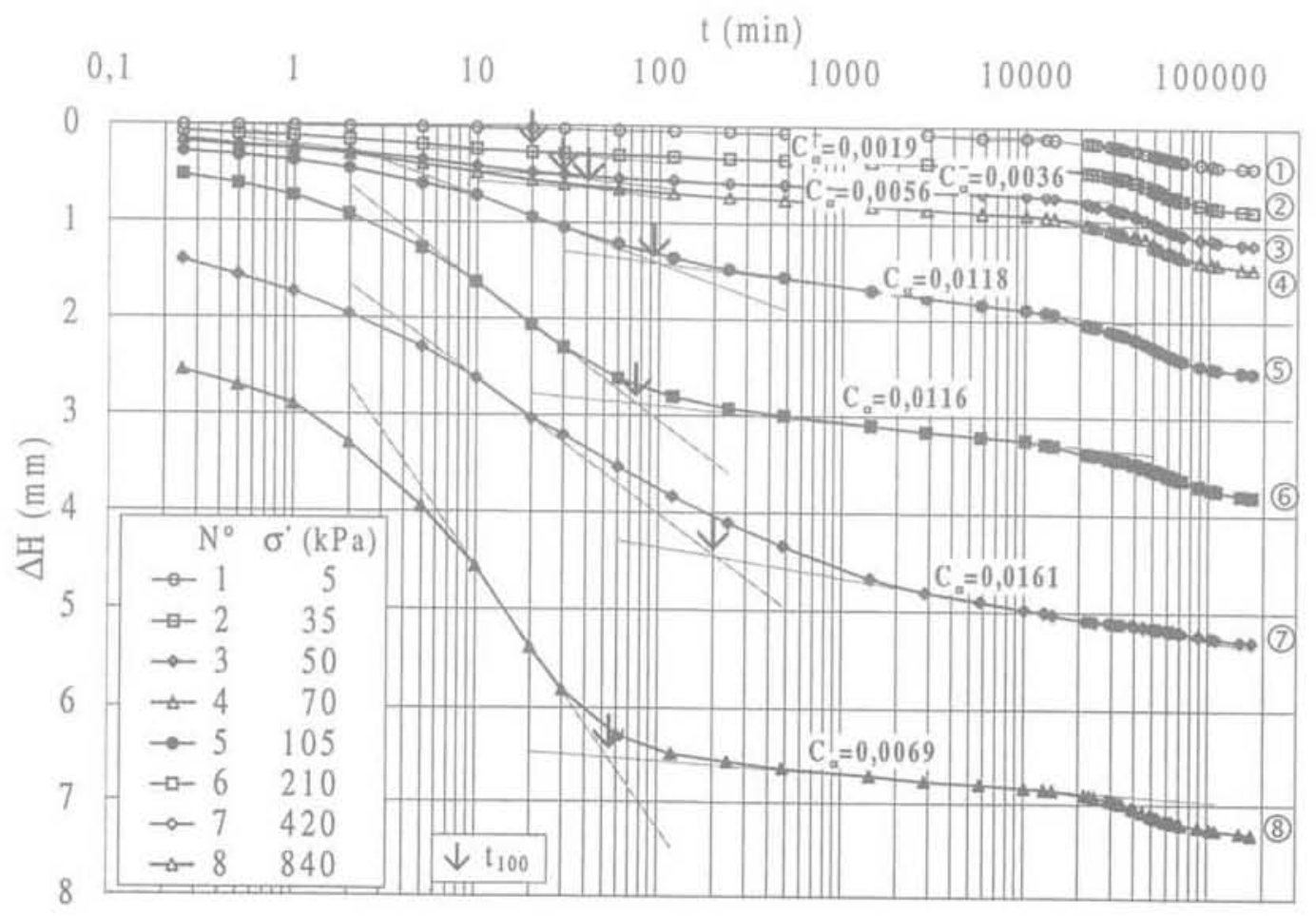

FiG,4 Résultats des essais de fluage œdométriques (8 essais de 300 jours). Oedometer creep test results ( 8 tests for 300 days). 
Les courbes de la figure 5, correspondant aux essais triaxiaux CIU de compression non drainée, atteignent leur maximum pour une valeur assez faible de la déformation axiale (de l'ordre de $2 \%$ au plus), ce qui est un signe de bonne qualité des éprouvettes testées, encore que des valeurs inférieures à $0,5 \%$ soient un meilleur gage de non remaniement dans tous les sols argileux naturels. L'absence de pic dans la plupart de ces courbes est par contre un témoignage du remaniement qu'ont dû subir les carottes d'argile lors de leur prélèvement au-delà de dix mètres de profondeur.
Les courbes de la figure 6 , correspondant aux essais triaxiaux $\mathrm{CK}$ U et $\mathrm{CK}$ IU, ont la même allure générale que les courbes de la figure 5 , avec des pentes initiales raides, conduisant au maximum du déviateur pour des valeurs de la déformation axiale inférieures à 2,5\%. La comparaison des courbes 2 et 6 avec les courbes 1 et 7 de la figure 5, qui correspondent à la même pression de consolidation de $40 \mathrm{kPa}$, montre que la reconsolidation sous les contraintes effectives en place a eu pour effet de diminuer le déviateur maximal (en moyenne $60 \mathrm{kPa}$ au lieu de $70 \mathrm{kPa}$ dans les essais CIU). Mais il
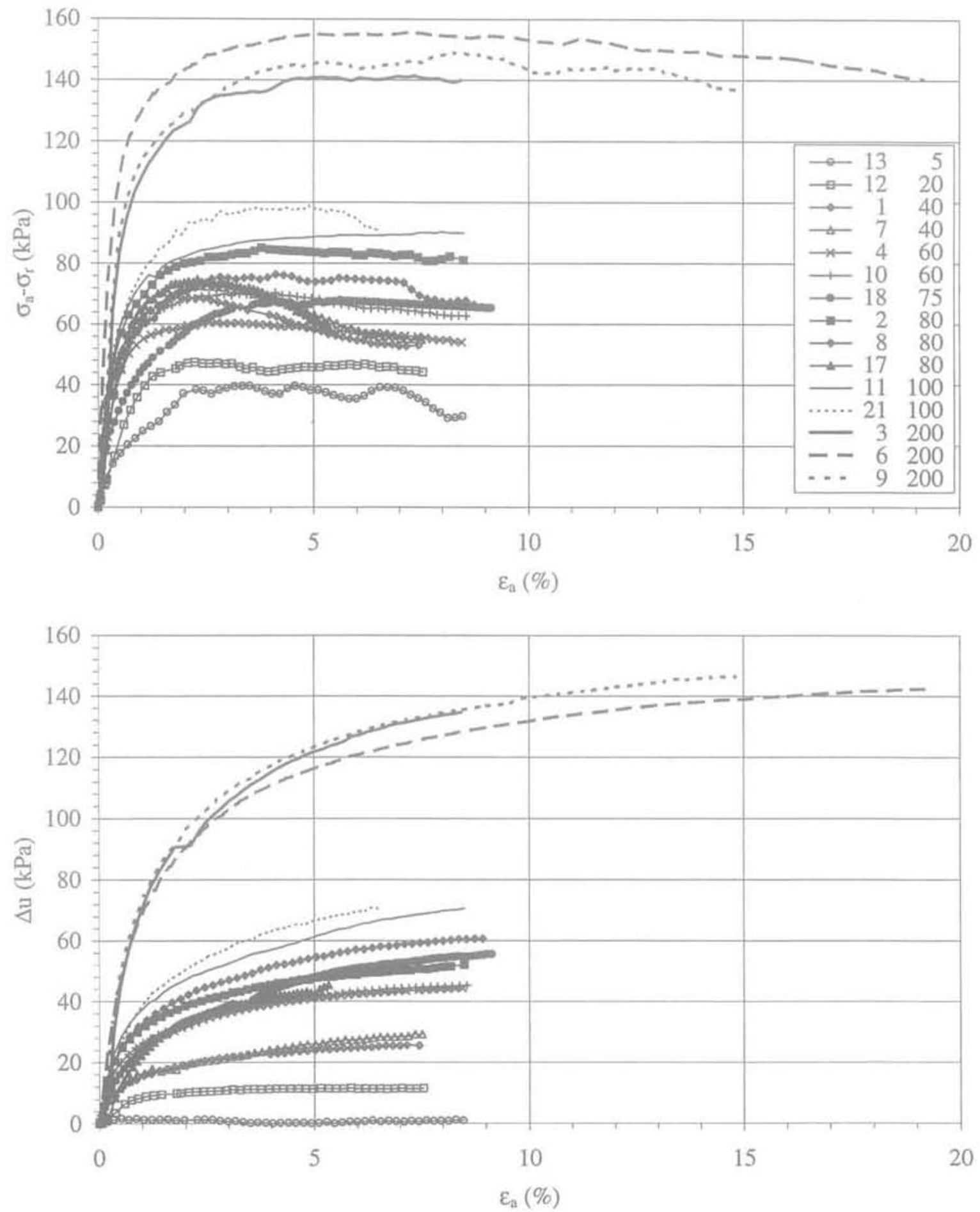

FiG.5 Résultats des essais triaxiaux CIU de compression non drainée sur des éprouvettes consolidées sous des contraintes isotropes différentes.

Results of CIU undrained triaxial compression tests on specimens consolidated at various isotropic stresses. 

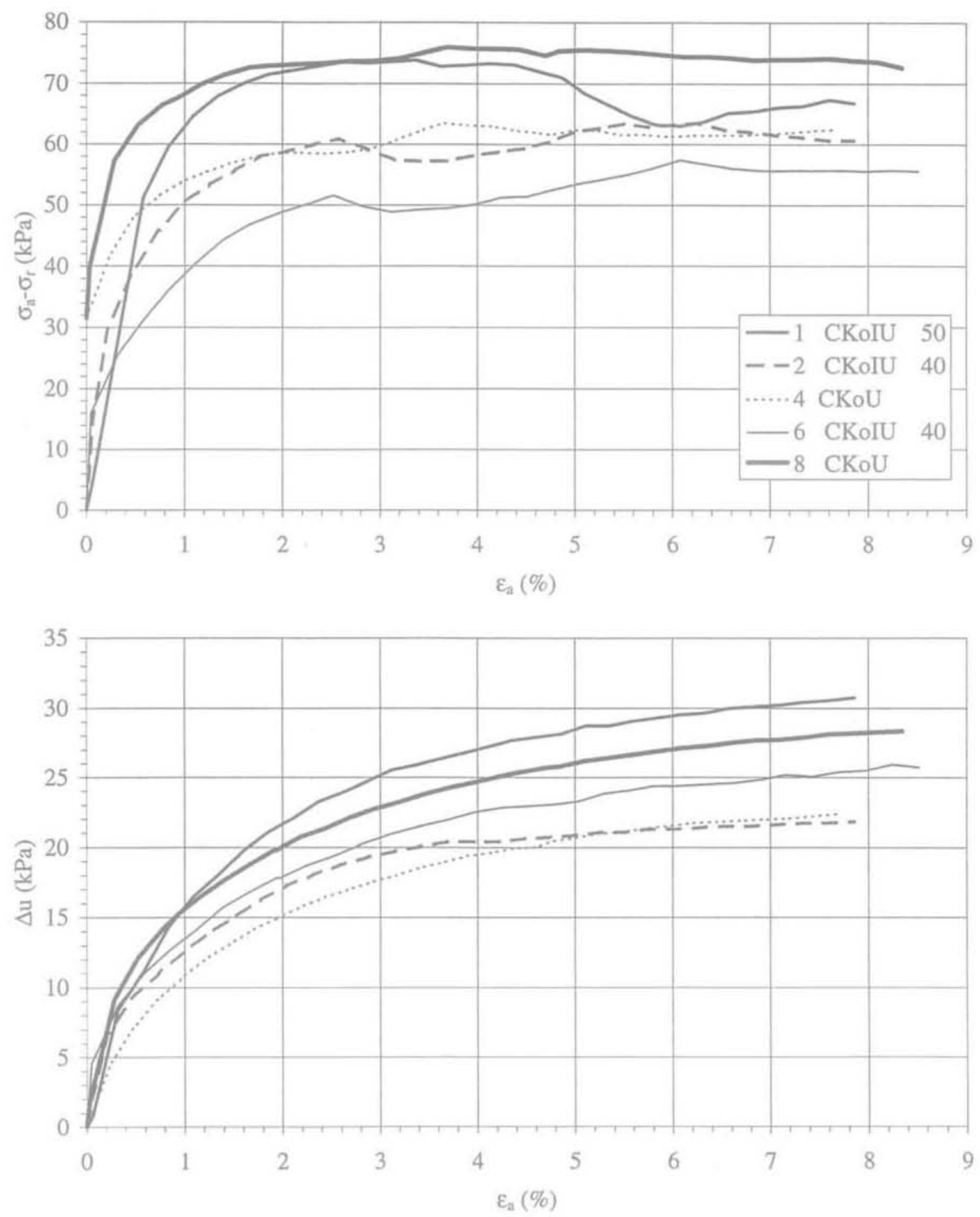

FG: 6 Résultats des essais triaxiaux $\mathrm{CK}_{\mathrm{o}} \mathrm{U}$ et $\mathrm{CK}_{\mathrm{o}} \mathrm{IU}$ de compression non dirainée sur des éprouvettes reconsolidées aux contraintes effectives en place $\left(\sigma_{\mathrm{vo}}^{\prime}, \mathrm{K}_{\mathrm{o}} \sigma_{\mathrm{vo}}^{\prime}\right)$ avec $\mathrm{K}_{\mathrm{o}}=0,55$.

Results of $\mathrm{CK}_{0} \mathrm{U}$ and $\mathrm{CK}_{\mathrm{o}} \mathrm{IU}$ undrained triaxial compression tests on specimens reconsolidated under the in situ effective stresses $\left(\sigma^{\prime}, K_{0}^{\prime} \sigma^{\prime}{ }_{v o}\right)$ with $K_{0}=0,55$.

s'agit peut-être d'un effet des difficultés rencontrées avec les engrenages de la presse utilisée pour ces essais.

Les résultats des essais triaxiaux EK $\mathrm{U}_{\text {et }} \mathrm{EK}_{0} \mathrm{IU}$ sont représentés sur la figure 7. Dans l'essai 1, exécuté après consolidation de l'éprouvette aux contraintes effectives en place puis sous une contrainte isotrope de $50 \mathrm{kPa}$, l'état d'équilibre est atteint rapidement et la déformation se poursuit à volume constant (pas de variation de la pression interstitielle). L'essai 4 tend vers la même limite, mais progressivement et avec augmentation de volume.
Les courbes de la figure 8 correspondent aux essais triaxiaux CID de compression drainée qui ont été exécutés pour caractériser l'anisotropie du comportement de l'argile : cinq essais sur éprouvettes taillées verticalement ( $1 \mathrm{~V}$ à $5 \mathrm{~V}$ ) et cinq essais sur éprouvettes taillées horizontalement $(1 \mathrm{H}$ à $5 \mathrm{H})$, reconsolidées sous pression moyenne isotrope égale à la contrainte effective des terres en place. Dans la plupart des essais, le déviateur des contraintes croit constamment avec la déformation axiale, jusqu'à des niveaux de déformations très importants (10 à $20 \%$ ). Les relations entre les déformations 


\section{$\varepsilon_{\mathrm{a}}(\%)$}

$-9$

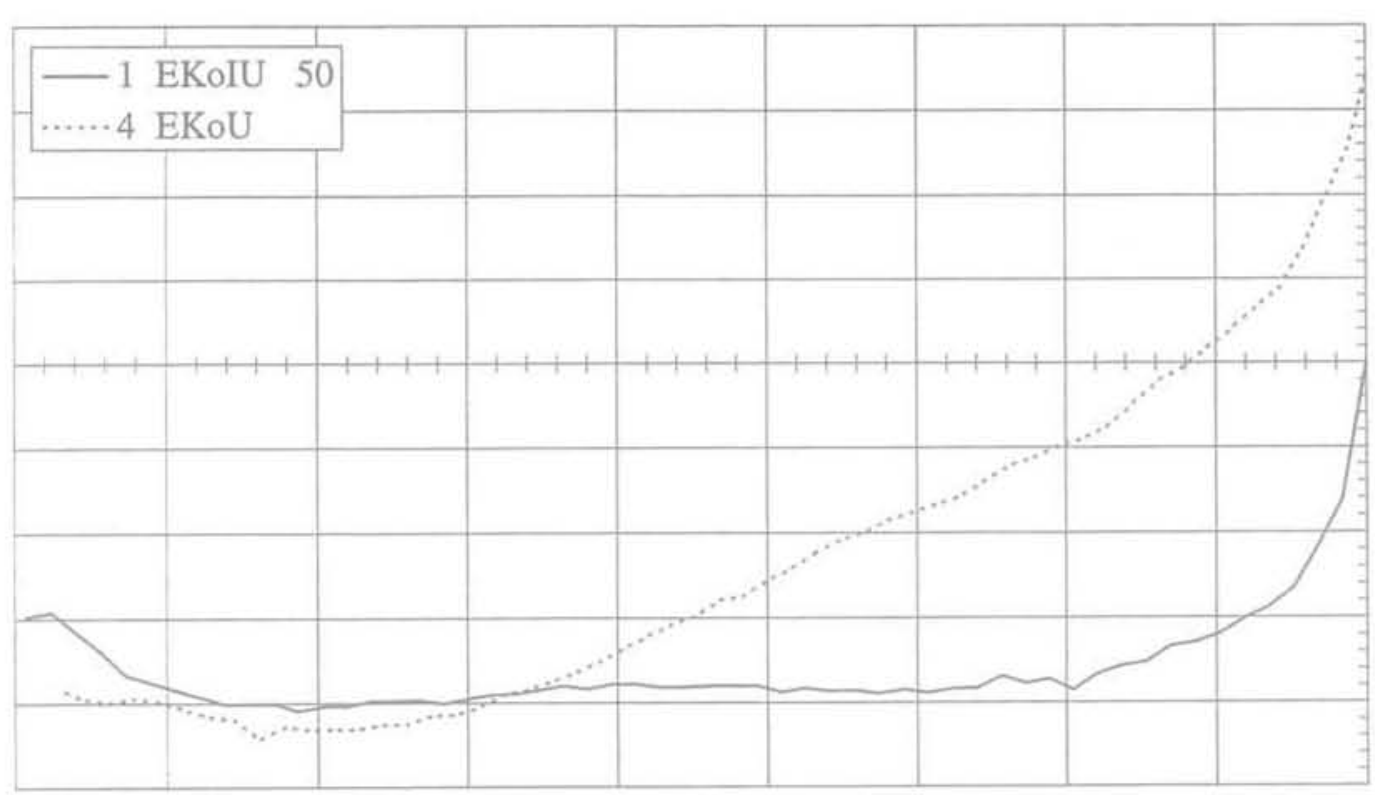

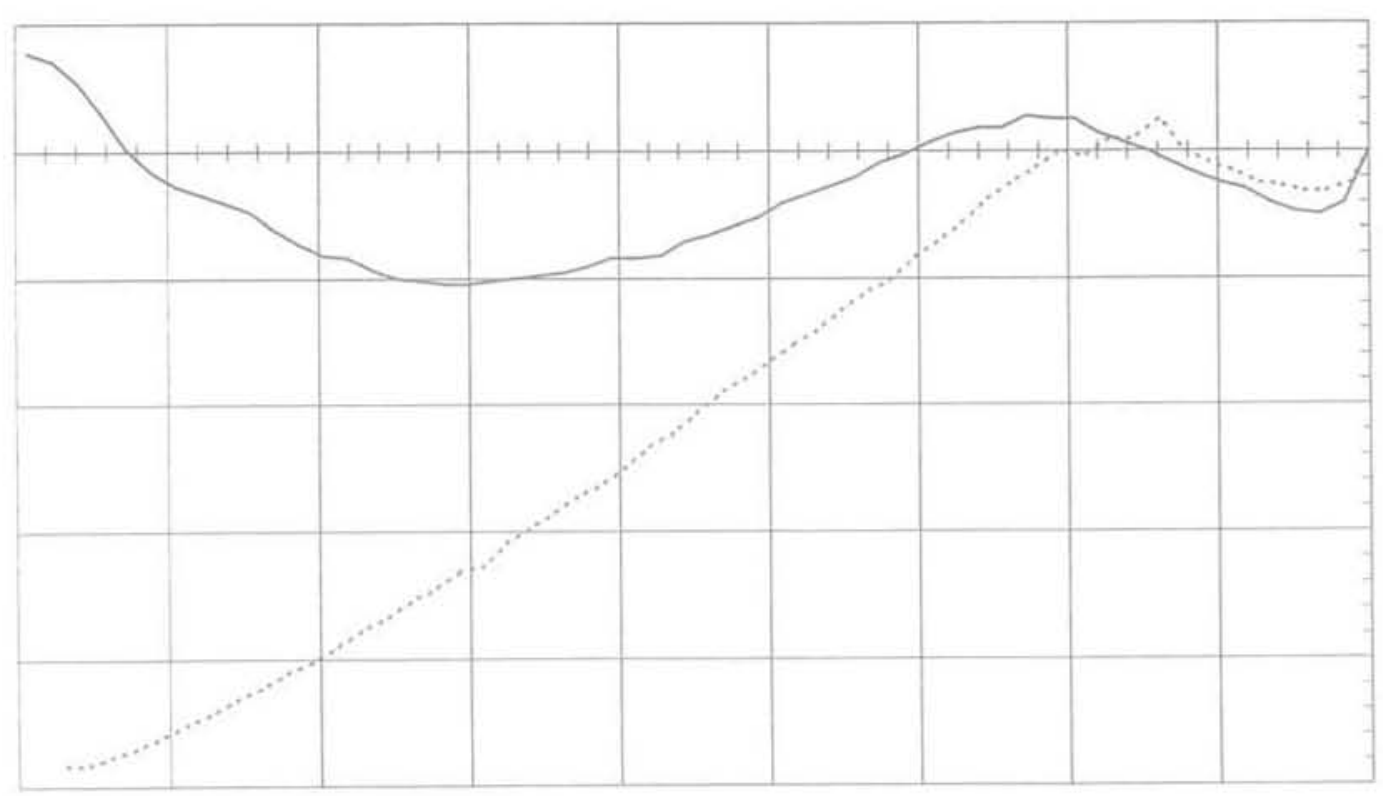

FIG.7 Résultats des essais triaxiaux EK U et EK IU d'extension non drainée sur des éprouvettes reconsolidées aux contraintes effectives en place $\left(\sigma^{\prime}{ }_{\mathrm{vo}}, \mathrm{K}_{\mathrm{o}} \sigma^{\prime}{ }_{\mathrm{vo}}\right)$ avec $\mathrm{K}_{\mathrm{o}}=0,55$.

Results of EK U and EK IU undrained triaxial extension tests on specimens reconsolidated under the in situ effective stresses $\left(\sigma_{\mathrm{w},}^{\prime}, \mathrm{K}_{0} \sigma_{\mathrm{ve}}^{\prime}\right)$ with $\mathrm{K}_{\mathrm{e}}=0,55$.

axiale et volumique sont peu différentes dans les deux cas. Mais on note que la pente initiale des courbes de variation du déviateur en fonction de la déformation axiale est plus élevée dans le cas des essais sur éprouvettes verticales. Par ailleurs, la forme des courbes de variation du déviateur des contraintes en fonction de la déformation axiale dans les deux cas et, notamment, l'absence de pic ou de palier de déviateur à des valeurs de la déformation axiale qui soient typiques d'éprouvettes non remaniées ou peu remaniées, fait que ces résultats devront être vérifiés sur d'autres éprouvettes plus intactes de ce sol.

On notera sur les courbes de la figure 9, correspondant aux essais triaxiaux CAP de consolidation anisotrope, que les mesures effectuées après 72 heures de consolidation donnent des points (notés 3j) éloignés de la courbe moyenne de consolidation, sans doute à cause des effets de compression secondaire, ce qui confirme l'influence de la durée des paliers de chargement appliqués aux éprouvettes sur la consolidation de l'argile. 

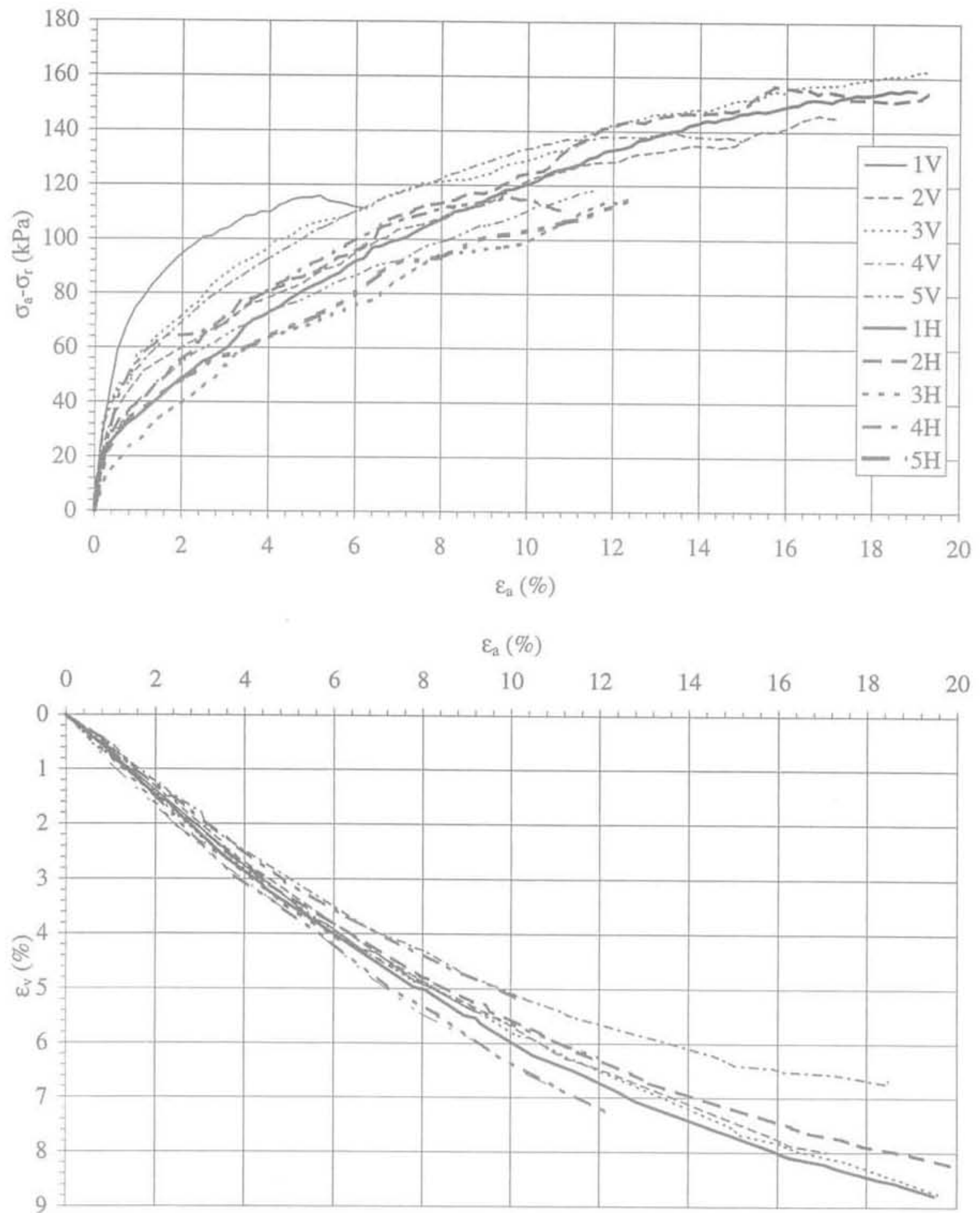

FlG. a Résultats des essais triaxiaux CID de compression drainée sur des éprouvettes reconsolidées sous une contrainte isotrope égale à la pression effective moyenne des terres en place (V : éprouvettes verticales, $\mathrm{H}$ : éprouvettes horizontales).

Results of CID drained triaxial compression tests on specimens consolidated isotropically under the in situ mean effective earth pressure ( $V$ : vertical specimens, $H$ : horizontal specimens). 


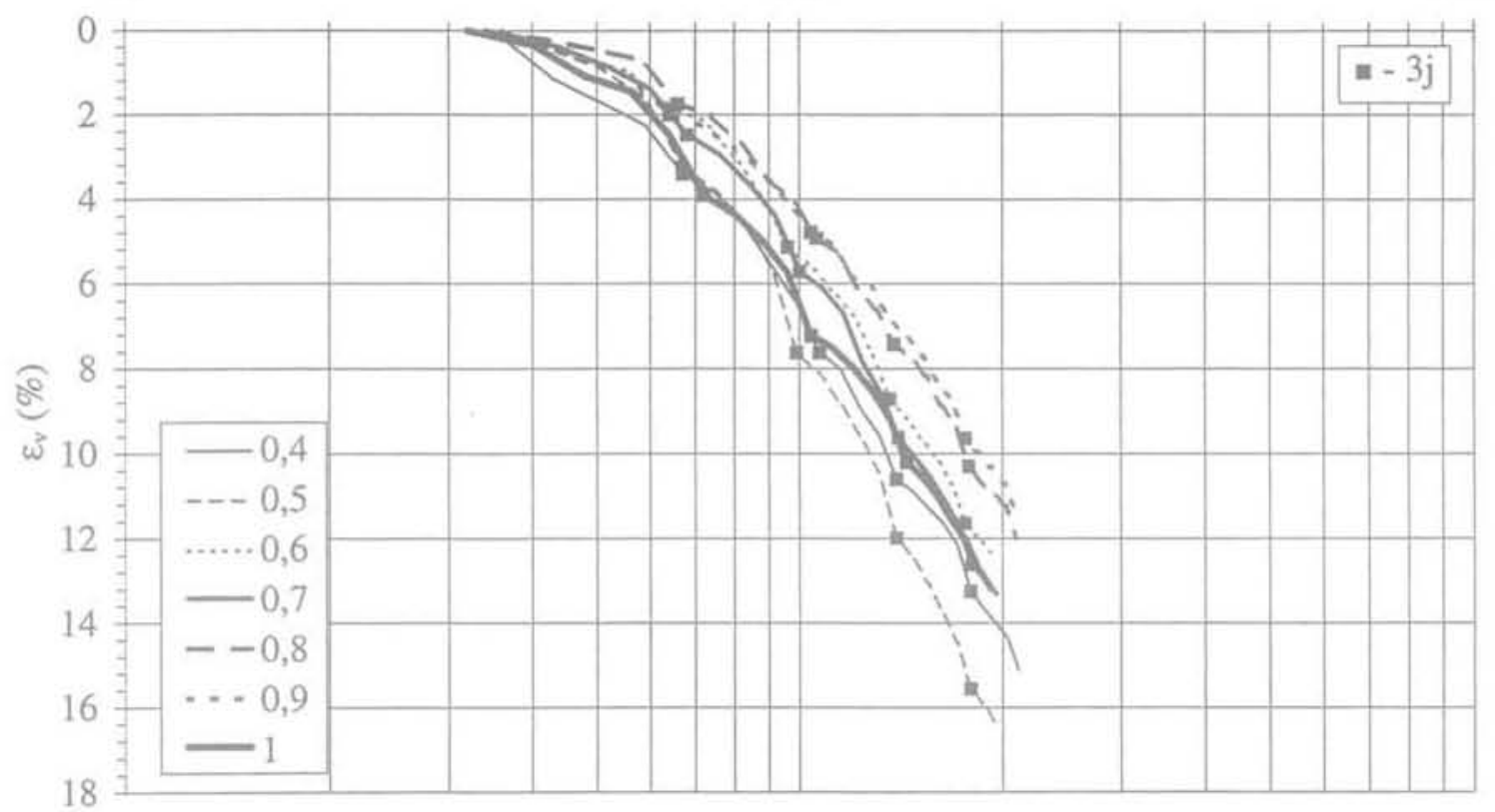

$\mathrm{q}(\mathrm{kPa})$

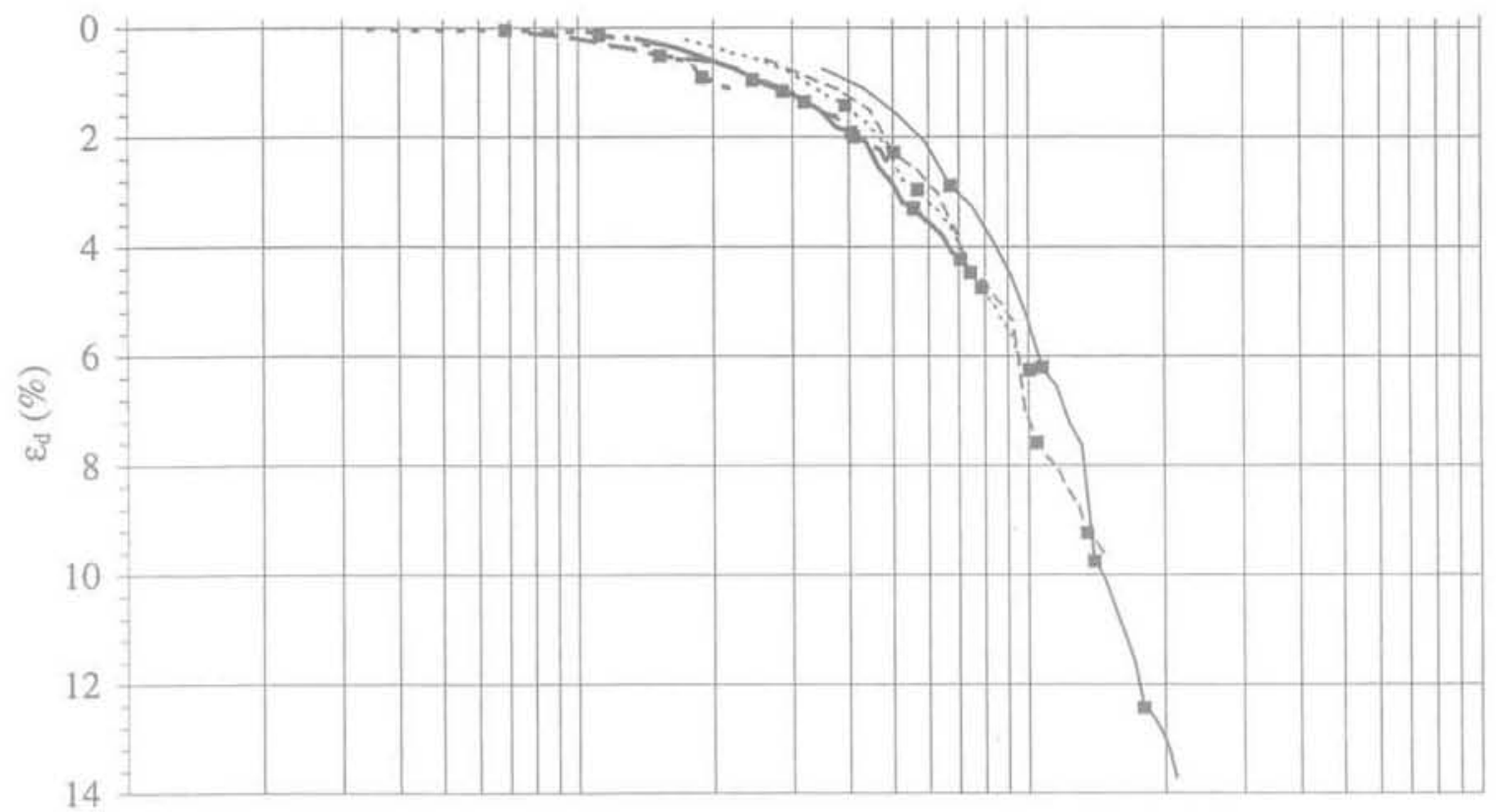

FG.9 Résultats des essais triaxiaux CAP de consolidation anisotrope sous des paliers de 24 heures (3j : points correspondant à des mesures effectuées après 72 heures de consolidation).

Results of CAP anisotropic consolidation triaxial tests under 24 hours stage loading ( $3 \mathrm{j}$ : points corresponding to measures carried out after 72 hours of consolidation).

\section{5}

\section{Interprétation des résultats}

\section{1}

\section{État limite}

Les chemins de contraintes effectives suivis dans les différents essais triaxiaux ont l'allure indiquée sur les figures 10 à 13. Si l'on admet, comme l'ont fait Tavenas et Leroueil (1979) pour l'argile de Saint-Alban et Magnan et al. (1982) pour l'argile de Cubzac-les-Ponts, que les points marquant le passage de l'argile de l'état surconsolidé à l'état normalement consolidé correspondent aux pics des courbes du déviateur des contraintes en fonction de la déformation axiale dans les essais de compression ou d'extension et aux coudes des courbes de déformation volumique en fonction de la contrainte moyenne effective dans les essais de consolidation anisotrope, on obtient les points représentés sur la figure 


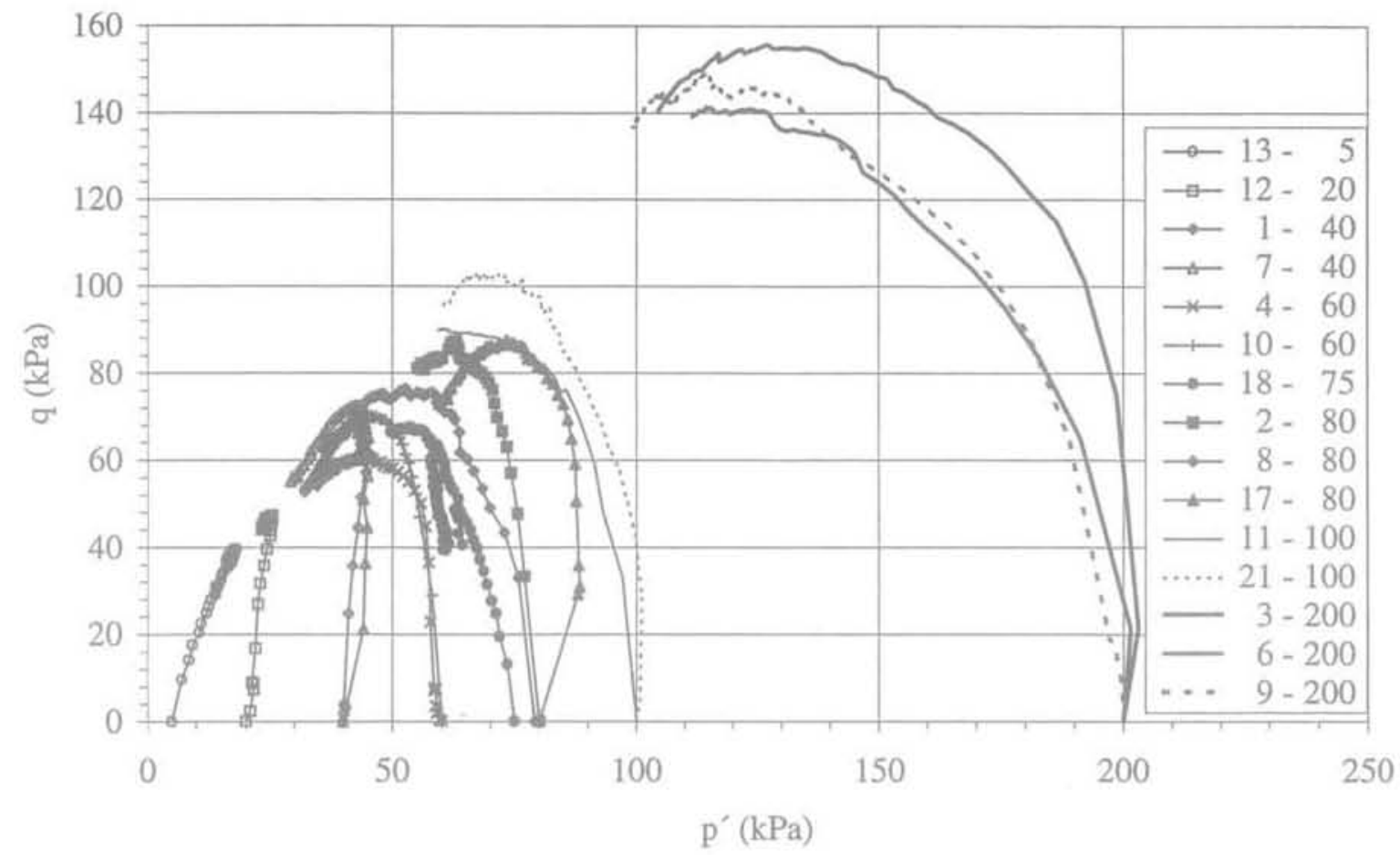

FG, 10 Chemins de contraintes effectives des essais triaxiaux CIU. Effective stress paths derived from CIU triaxial tests.

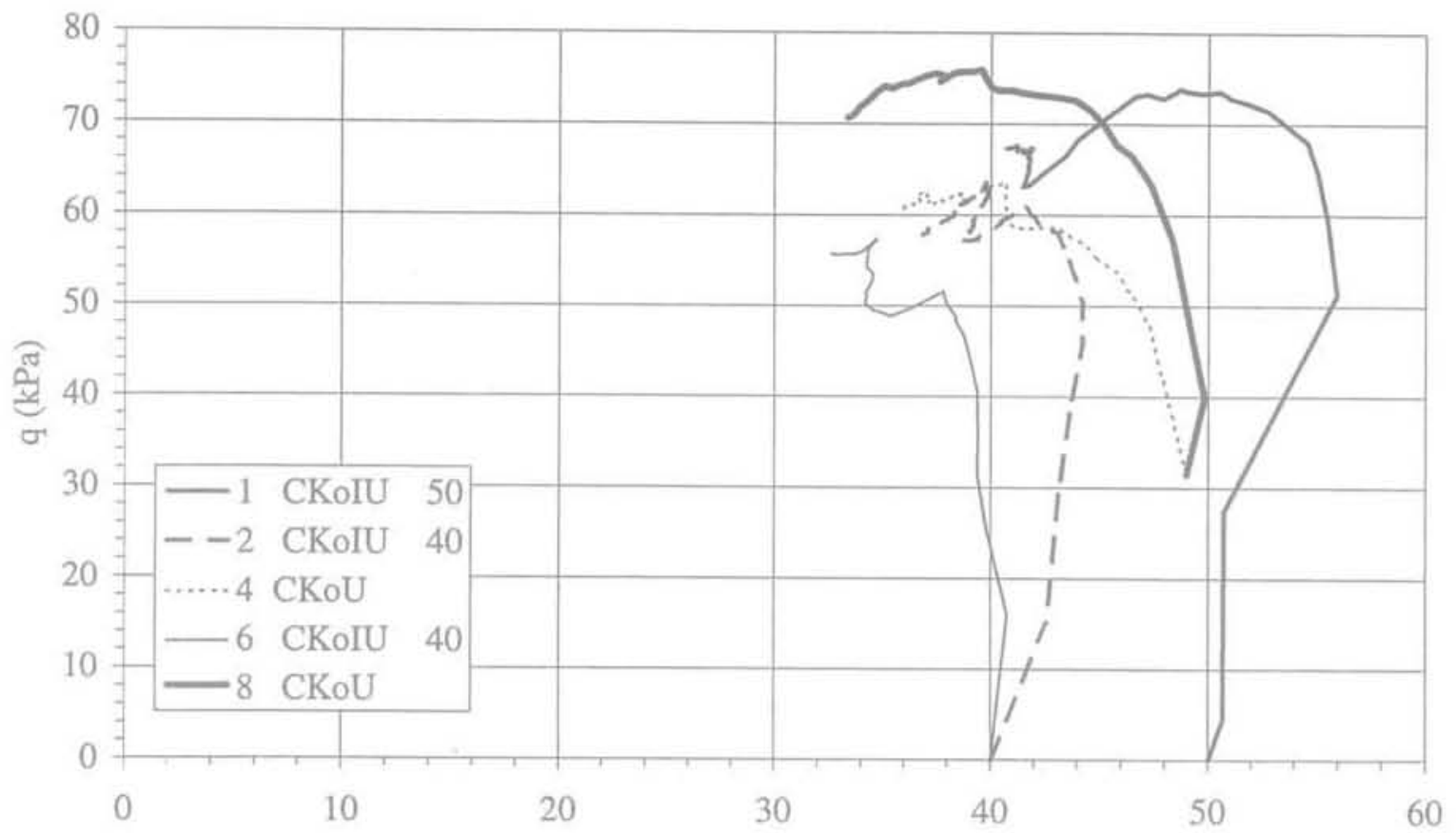

FG.11 Chemins de contraintes effectives des essais triaxiaux CK $U$ et CK IU. Effective stress paths derived from $\mathrm{CK}_{0} \mathrm{U}$ and $\mathrm{CK}_{0} \mathrm{IU}$ triaxial tests.

14a dans le plan $\left(\mathrm{s}^{\prime}-\mathrm{t}\right.$ ) et sur la figure $14 \mathrm{~b}$ dans le plan $\left(\sigma^{\prime} / \sigma_{p}^{\prime}-t / \sigma_{p}^{\prime}\right)$, où $\sigma_{p}^{\prime}$ désigne la pression de préconsolidation de l'argile. Ces points définissent une première allure de la courbe d'état limite de l'argile de Guiche et de la droite de Mohr-Coulomb, qui correspond aux conditions de rupture en dehors du domaine surconsolidé initial. La normalisation du système de coordonnées a été effectuée pour chaque éprouvette par rapport à la pression de préconsolidation $\sigma_{p}^{\prime}$ de l'argile, déduite des essais de compressibilité œedométrique exécutés à la même profondeur. Les valeurs de $\sigma^{\prime}$ utilisées sont indiquées dans le tableau III. Cette première ébauche de la courbe d'état limite a ensuite été corrigée, pour tenir compte des effets du temps et de la profondeur des éprouvettes testées.

Pour poursuivre l'étude de la courbe d'état limite, nous avons « normalisé » les coordonnées de la figure 14a par rapport à la pression de préconsolidation de 
$\mathrm{p}^{\prime}(\mathrm{kPa})$

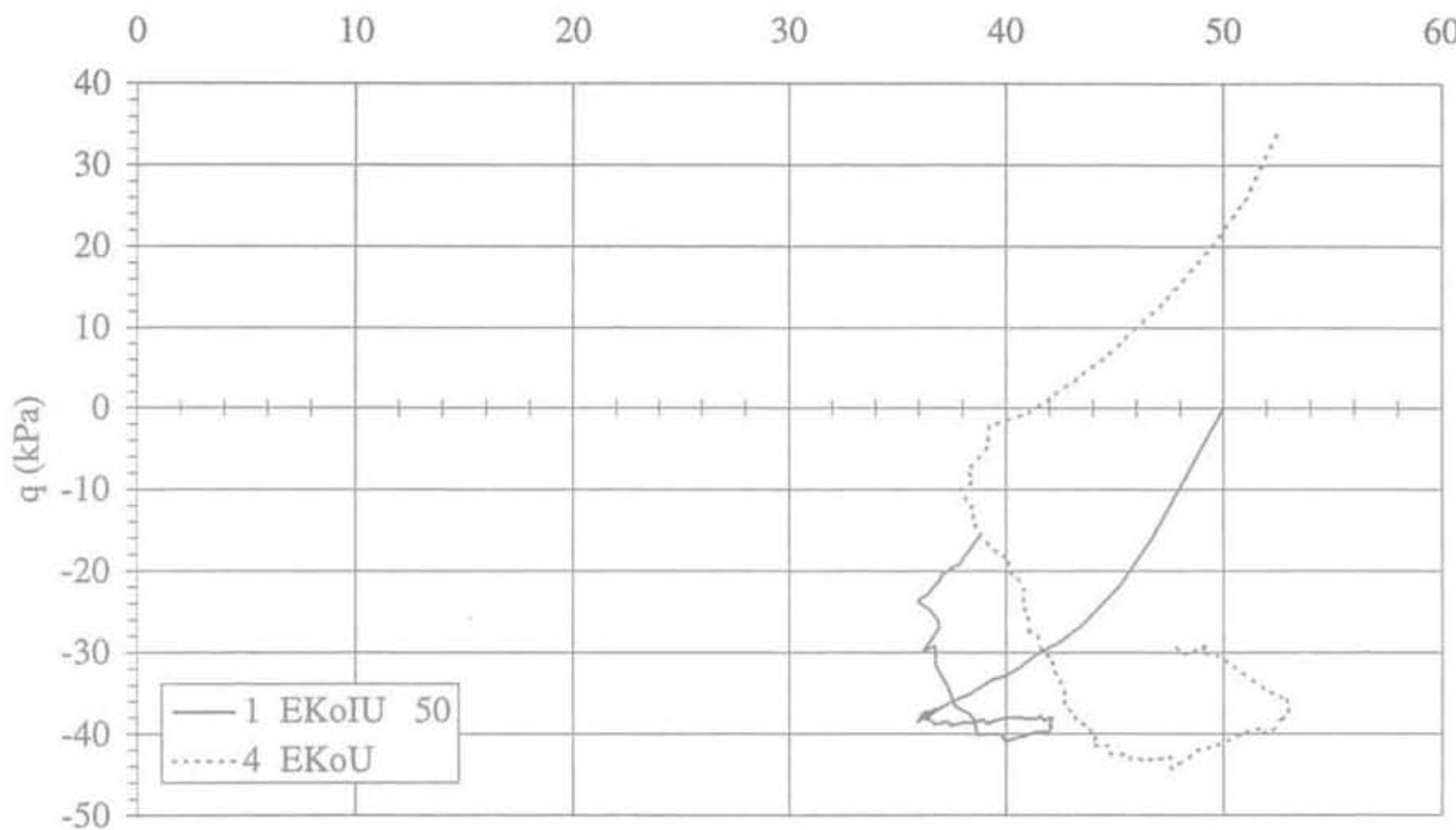

FG 12 Chemins de contraintes effectives des essais triaxiaux EK U et EK IU. Effective stress paths derived from $\mathrm{EK}_{0} \mathrm{U}$ and $E \mathrm{~K}_{0} \mathrm{IU}$ triaxial tests.

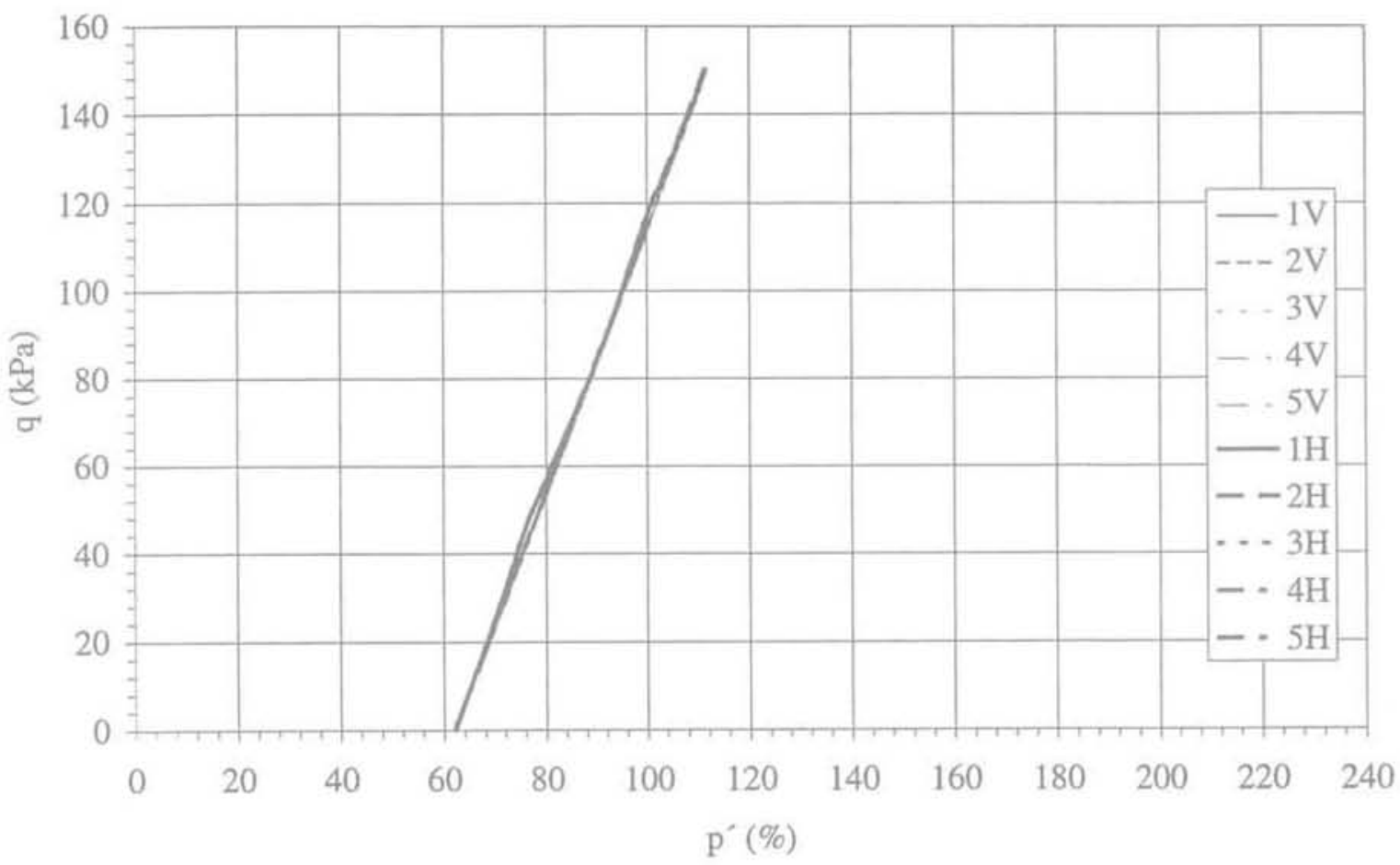

Fic. 13 Chemins de contraintes effectives des essais triaxiaux CID.

Effective stress paths derived from CID triaxial tests.

référence $\left(\sigma_{p}^{\prime}\right)_{\text {rér }}$ calculée au niveau de chaque éprouvette testée, en tenant compte cette fois de la vitesse d'application de la charge dans l'essai triaxial (tableau III). Les coefficients correcteurs pour les vitesses d'essai ont été déduits de la loi de fluage décrite plus loin, en rapportant toutes les pressions de préconsolidation $\sigma_{p}^{\prime}$ à une vitesse de déformation com- mune $\varepsilon_{0}=6 \cdot 10^{-8} \mathrm{~S}^{-1}$. On obtient ainsi les points représentés sur la figure 15.

La courbe d'état limite de référence représentée sur la figure 15 peut être décrite de façon approximative par une ellipse ayant pour axe principal la droite ( $\mathrm{K}_{0} »$, caractérisant l'état des contraintes effectives de l'argile en place, et calée sur les points expérimentaux situés 

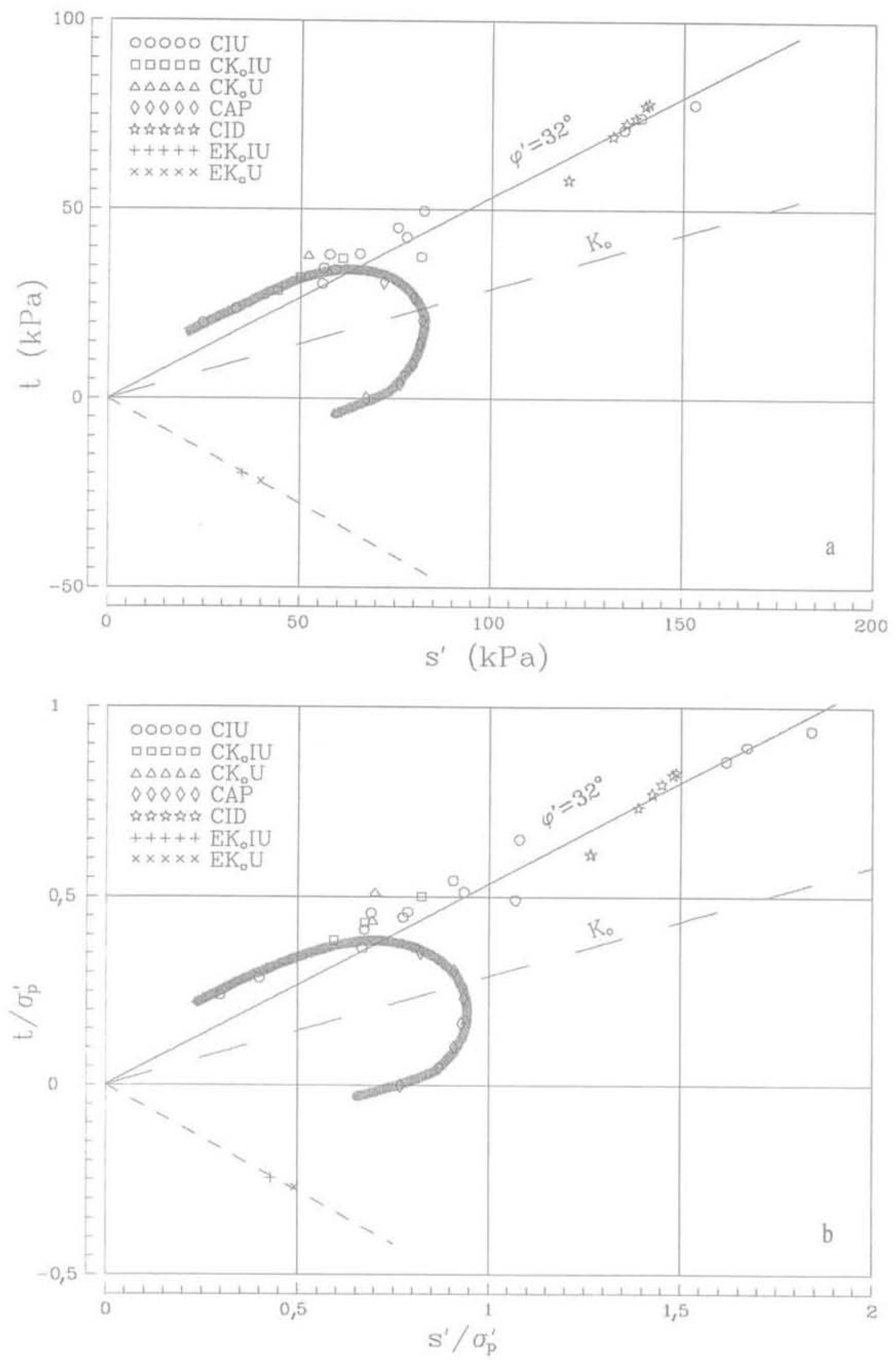

FG. 14 Courbe d'état limite de l'argile de Guiche $\left[a /\right.$ plan $\left(s^{\prime}-t\right) ; b /$ plan $\left.\left(s^{\prime} / \sigma_{p}^{\prime}-t / \sigma^{\prime}\right)\right]$ (première ébauche). Limite state curve of the Guiche clay $\left[a /\left(s^{\prime}-t\right)\right.$ diagram; $b /\left(s^{\prime} / \sigma_{p}-t / \sigma_{p}^{\prime}\right)$ diagram $]$ (first trial).

16

REVUE FANCAISE DE GEOTEOHACUE

N+B1
4F thinestre $199 ?$ 


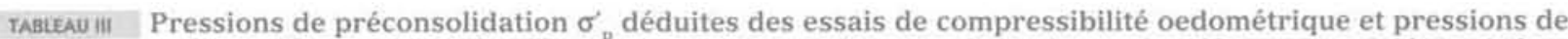
préconsolidation de référence $\left(\sigma_{0}^{p}\right)_{\text {réf }}$ calculées en fonction de la vitesse de déformation $\varepsilon$ déterminée pour chaque essai triaxial.

Preconsolidation pressure $\sigma^{\prime}$ derived from oedometer compression tests and reference preconsolidation pressure $\left(\sigma_{p}^{\prime}\right)_{\text {irf }}$ computed versus strain rate $\dot{\varepsilon}$ determined for each triaxial test.

\begin{tabular}{|c|c|c|c|c|c|}
\hline Séries & & Essais & $\sigma_{p}^{t}(\mathrm{kPa})$ & $\varepsilon\left(s^{-1}\right)$ & {$\left[\sigma_{p}^{\circ}\right)_{\text {mar }}(\mathrm{kPa})$} \\
\hline \multirow[t]{2}{*}{$\mathrm{T} 3$} & \multirow[t]{2}{*}{$\mathrm{CIU}$} & 1 à 13 & 83,2 & $1,32.10^{-6}$ & 99,4 \\
\hline & & 14 à 21 & 76,2 & $1,32 \cdot 10^{-7}$ & 88,5 \\
\hline T4 & $\mathrm{CK}_{\mathrm{n}} \mathrm{U}$ & 3 et 8 & 74,2 & $1,32.10^{-4}$ & 99,4 \\
\hline 15 & $\mathrm{CK}_{n} \mathrm{IU}$ & 1 à 3 et 5 à 7 & 74,2 & $1,32.10^{-11}$ & 99,4 \\
\hline TE & $E_{0} \mathrm{U}$ & 4 & 81,4 & $1.32 .10^{-5}$ & 99,4 \\
\hline 17 & $\mathrm{EK}_{\mathrm{c}} \mathrm{IU}$ & 1 à 3 & 87,6 & $1,32.10^{-4}$ & 99,4 \\
\hline $\mathrm{T} 8$ & CAP & 1 à 7 & 87,6 & $1,94,10^{-7}$ & 90,2 \\
\hline T9 & CID & $1 \mathrm{~V}$ à $5 \mathrm{~V}$ et $1 \mathrm{H}$ à $5 \mathrm{H}$ & 94,8 & $1.32 .10^{-1}$ & 88,5 \\
\hline
\end{tabular}

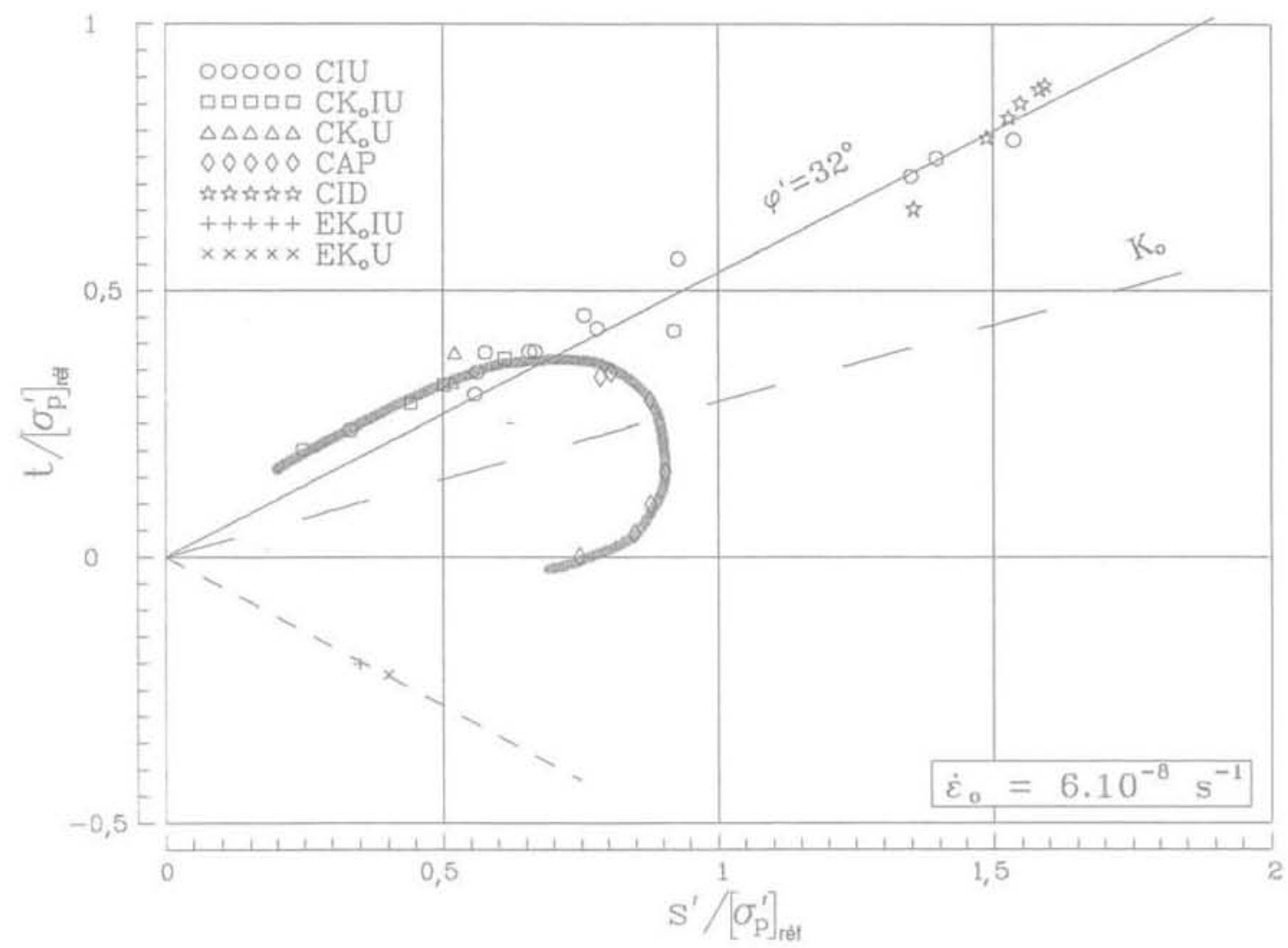

FIG.15 Courbe d'état limite de référence de l’argile de Guiche. Reference limit state curve of Guiche clay.

en dessous de la droite de Mohr-Coulomb. Pour sa part, la droite de Mohr-Coulomb tracée à l'extérieur de la courbe d'état limite en passant par l'origine des coordonnées a une pente $\operatorname{tg} \theta=0,54$, qui correspond à un angle de frottement interne effectif $\varphi^{\prime}=32^{\circ}$ pour une cohésion effective $c^{\prime}=0 \mathrm{kPa}$.

Les composantes de l'incrément de déformation plastique dans les essais triaxiaux de consolidation anisotrope ont été déterminées par construction graphique sur les courbes $\left.\alpha \varepsilon_{y}-\operatorname{lgp} p^{\prime}\right)$ et ( $\varepsilon_{\mathrm{d}}-\operatorname{lgq} »$ de chaque essai, au point d'état limite, La construction effectuée vise à enlever de la déformation totale mesurée la déformation élastique (obtenue par extrapolation de la déformation avant l'état limite) correspondant à un incrément de contrainte effective moyenne donné. Ces composantes, transformées conformément au système de représentation $\left(\mathrm{s}^{\prime}-\mathrm{t}\right)$ adopté ici (demi-somme $\varepsilon^{p+}=\left(\varepsilon^{p}+\varepsilon^{p}\right) / 2$ et demi-différence $\left.\varepsilon^{p-}=\left(\varepsilon^{p}-\varepsilon^{p}\right) / 2\right)$, permettent de calculer la direction des vecteurs de déformation plastique en chaque point d'état limite, qui sont représentés sur la figure 16. 
On observe que les vecteurs de déformation plastique ne sont pas normaux à la courbe d'état limite, la loi d'écoulement n'est donc pas associée. Dans la partie supérieure de la courbe d'état limite, correspondant à la rupture des éprouvettes, il est plus difficile de définir la loi d'écoulement plastique. La première difficulté est liée à l'apparition très fréquente d'une surface de rupture sur laquelle se concentre la déformation de l'éprouvette au-delà d'un certain niveau de chargement. Cette " localisation de la déformation $»$ interdit d'interpréter la déformation ultérieure de l'éprouvette comme celle d'un milieu continu à déformation élastoplastique homogène. Dans le programme d'essajs réalisés sur l'argile de Guiche, aucune information n'a été obtenue sur les déformations sur cette partie de la courbe d'état limite.

\section{2}

\section{Anisotropie}

L'anisotropie du comportement de l'argile de Guiche a été analysée sur la base des essais triaxiaux CID de compression drainée réalisés sur des éprouvettes taillées verticalement et horizontalement dans la même carotte. Les figures 17 et 18 présentent respectivement la partie initiale des courbes d'évolution du déviateur des contraintes et de la déformation volumique en fonction de la déformation axiale. L'analyse de ces courbes appellent les remarques suivantes:

- on peut déterminer les modules d'Young dans les directions verticale et horizontale, qui sont plus élevés dans la direction verticale que dans la direction horizontale, d'un facteur voisin de deux. Les modules déterminés entre l'origine des coordonnées et le premier point de mesure sont environ trois fois plus forts que ceux que l'on calcule pour l'intervalle de déformation axiale de 0,25 à $0,75 \%$;

- outre les modules d'Young vertical $E_{y}^{\prime}$ et horizontal $E_{n}^{\prime}$, on peut déduire des essais réalisés les valeurs des coefficients de Poisson $v_{\text {vh }}^{\prime}$ et $v_{h+1}^{\prime}$;

- le coefficient de Poisson $v_{\text {yh }}^{\prime}$ se déduit de la pente $\left[\mathrm{d} \varepsilon_{/} / \mathrm{d} \varepsilon_{\mathrm{a}}\right]_{\mathrm{y}}$ des courbes de variation de la déformation volumique $e_{u}$ en fonction de la déformation axiale $\varepsilon_{\text {a }}$ dans les essais exécutés sur les éprouvettes verticales :

- le coefficient de Poisson $v^{\prime}$ se déduit de la pente $\left[\mathrm{d} \varepsilon_{/} / \mathrm{d} \varepsilon_{\mathrm{\mu}}\right]_{\mathrm{H}}$ des courbes de variation de la déformation volumique $\varepsilon_{\mathrm{v}}$ en fonction de la déformation axiale $\varepsilon_{\mathrm{u}}$ dans les essais exécutés sur les éprouvettes horizontales et du rapport $\mathrm{n}=\mathrm{E}_{\mathrm{n}}^{\prime} / \mathrm{E}_{\mathrm{v}}^{\prime}$ des valeurs des modules d'Young déterminés auparavant.

Les plages de variation et les valeurs moyennes des modules d'Young vertical et horizontal et des coefficients de Poisson sont rassemblées dans le tableau IV. Les essais réalisés ne permettent pas de déterminer directement la valeur du module de Coulomb dans un plan vertical $G^{\prime}$, qui nécessite des moyens d'essai différents (Meftah, 1988).

On notera une dispersion notable des valeurs de chaque paramètre dans les deux sens vertical et horizontal et sans relation apparente avec la teneur en eau de l'argile, dont les valeurs sont relativement peu variables d'une éprouvette à I'autre. Néanmoins, compte tenu des conditions expérimentales, d'une part, et du matériau lui-même (effet de la profondeur sur l'état des carottes), d'autre part, on peut penser que les valeurs sont en moyenne représentatives du compor- tement anisotrope de l'argile à l'état surconsolidé, c'està-dire à l'intérieur de sa courbe d'état limite. On peut toutefois noter que :

- les valeurs relativement faibles de certains modules d'Young et les déformations importantes des éprouvettes avant la rupture laissent craindre un certain remaniement du sol avant les essais;

- les coefficients de Poisson ne violent pas le critère de l'énergie potentielle, ce qui laisse penser que les essais drainés permettent d'obtenir des valeurs cohérentes des paramètres d'aélotropie des sols argileux. En revanche, ces valeurs ne vérifient pas la relation établie par Atkinson (1975) et vérifiée par lui sur l'argile de Londres, dans laquelle il considère que deux éprouvettes d'argile découpées l'une dans la direction de l'axe d'isotropie et l'autre dans la direction perpendiculaire à cet axe sont caractérisées par la même dilatance lorsqu'elles sont sollicitées en compression à partir d'un état de contraintes isotrope.

On constate par ailleurs que ces valeurs sont voisines des résultats trouvés sur d'autres argiles naturelles comparables à l'état surconsolidé.

\section{Compressibilité}

La détermination de la pression de préconsolidation $\sigma_{p}^{\prime}$ et des indices de compression $C_{c}$ et de gonflement $\mathrm{C}_{\mathrm{s}}^{\mathrm{p}}$ a été effectuée conformément à la procédure d'interprétation décrite dans les méthodes d'essai LPC. (Magnan et al., 1985). Les résultats sont regroupés sur la figure 19, avec les autres résultats tirés des essais œdométriques (paramètres physiques, paramètres de perméabilité). Sur cette figure, les valeurs des paramètres sont représentées à leur profondeur de mesure. Les trois premiers niveaux de mesure correspondent à des éprouvettes prélevées au carottier à piston stationnaire de $100 \mathrm{~mm}$ de diamètre. Les quatre niveaux inférieurs correspondent à des prélèvements au carottier de 200 mm de diamètre.

L'analyse globale des résultats conduit à déclarer remaniés et non représentatifs les résultats obtenus à $11,7 \mathrm{~m}$ de profondeur, au motif que les pressions de préconsolidation $\sigma^{\prime}$ sont beaucoup trop faibles, avec des rapports de surconsolidation $\mathrm{R}_{\text {ci }}<1$, et les indices de compression $C$ aussi. Cette observation est conforme à l'expérience que nous avons acquise sur les performances des carottiers à piston stationnaire dans ce type de sol: au-delà d'une dizaine de mètres de profondeur, les carottes prélevées sont en général remaniées, quelles que soient les précautions prises lors du prélèvement. Pour les autres profondeurs, notamment au-delà de $12 \mathrm{~m}$ sous la surface du terrain naturel, la surconsolidation très faible traduit une certaine perturbation des éprouvettes testées, mais cette situation a été jugée acceptable pour la poursuite de l'étude. Il faut toutefois garder à l'esprit que les résultats des essais triaxiaux fournissent sans doute une estimation par défaut de la résistance et des modules de l'argile de Guiche. Une estimation vraisemblable de la pression de surconsolidation sur le site de Guiche, dans la couche d'argile molle étudiée ici, peut être tirée des valeurs maximales déduites des essais aux différentes profondeurs: environ $10 \mathrm{kPa}$ de plus que la contrainte effective verticale initiale $\sigma^{\prime}$. 


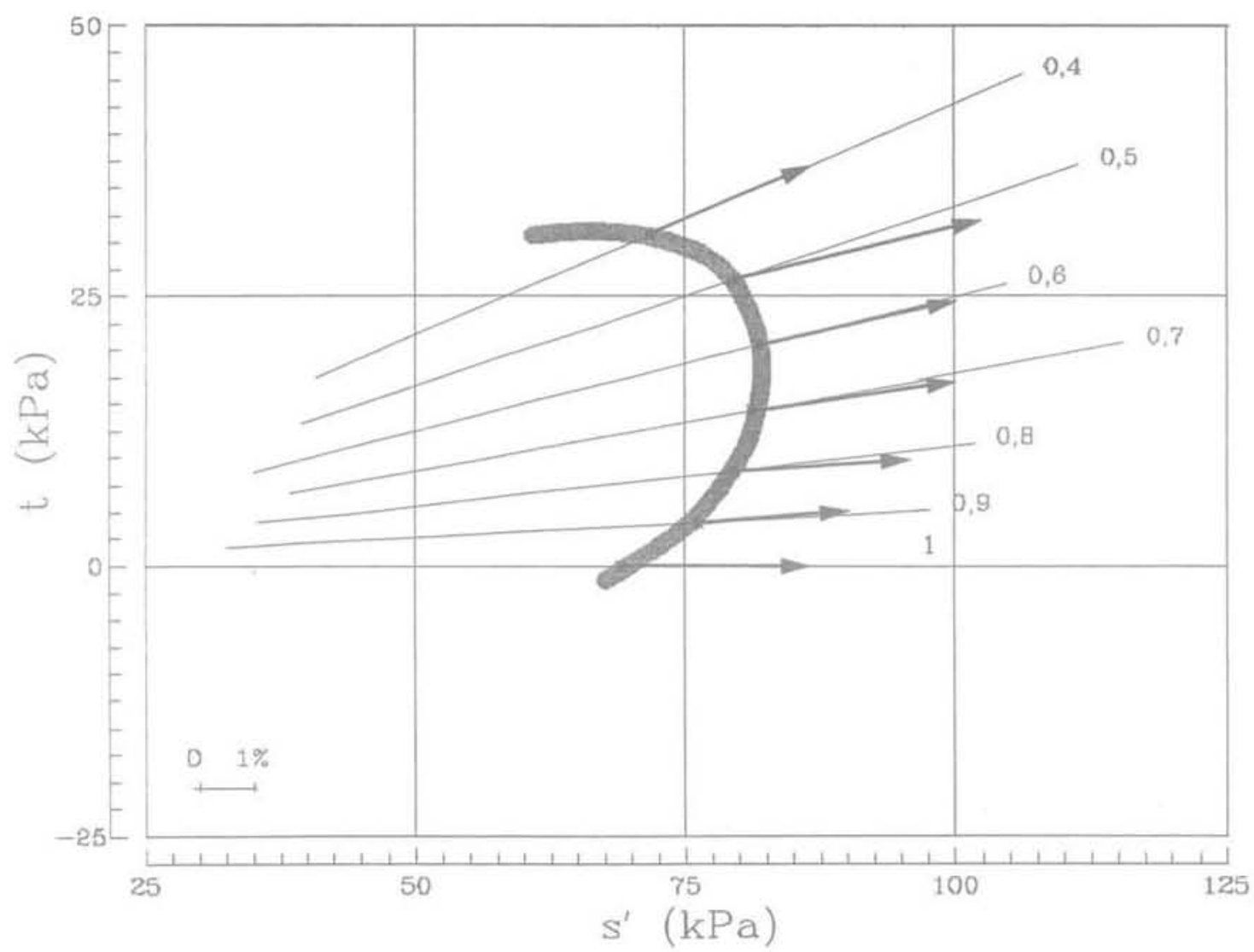

FIG. 18 Vecteurs de déformation plastique aux points d'état limite appartenant à la zone des contraintes d'écrouissage.

Vectors of plastic deformation along the limit state curve in the stress hardening area.

TABLEAU IV Plages de variation et valeurs moyennes des modules d'Young et des coefficients de Poisson. Variation ranges and mean values of Young's modulus and Poisson's ratios.

\begin{tabular}{|c|c|c|c|c|}
\hline Parametres & $\mathrm{E}_{v}^{\prime}(\mathrm{MPa})$ & $\mathrm{E}_{\mathrm{K}}^{\prime}(\mathrm{MPa})$ & $v_{v h}^{\prime}$ & $v_{\text {fin }}^{\prime}$ \\
\hline Plages de variation & $16,3-22,8$ & $7,6-12,4$ & $0,11-0,19$ & $0,09-0,32$ \\
\hline Moyennes & 18,3 & 9,9 & 0,14 & 0,21 \\
\hline
\end{tabular}

Une fois éliminés les résultats obtenus à $11.7 \mathrm{~m}$ de profondeur, on obtient les valeurs moyennes représentatives suivantes pour les caractéristiques de compressibilité de l'argile de Guiche entre 10 et $15 \mathrm{~m}$ de profondeur :

- indice des vides en place $\varepsilon_{0}=1,6$

- indice de compression $\mathrm{C}_{\mathrm{c}}=0,8$;

- indice de gonflement $\mathrm{C}_{\mathrm{s}}=0,07$;

- pression de préconsolidation $\sigma_{\mathrm{p}}^{\prime}=\sigma_{\mathrm{vo}}+10 \mathrm{kPa}$.

\section{4}

\section{Perméabilité}

Les courbes de consolidation sous les différentes charges appliquées aux éprouvettes ont été interprétées pour en déduire les coefficients de consolidation $\mathrm{c}_{\mathrm{v}}$, puis les coefficients de perméabilité verticale $\mathrm{k}_{\mathrm{v}}$ compte tenu des déformations observées pendant la consolidation primaire sous l'incrément de charge appliqué et des modules œedométriques $E_{\text {oed }}$ correspondants.

La procédure d'analyse utilise principalement la méthode de Taylor (variation de l'indice des vides en fonction de la racine carrée du temps), telle qu'elle est décrite par les méthodes d'essai LPC (Magnan et al., 1985), mais s'appuie en parallèle sur la construction de Casagrande (variation de l'indice des vides en fonction du logarithme du temps) pour contrôler que la déformation à $t_{00}$ correspond bien à environ $90 \%$ de la déformation totale pendant la consolidation primaire. Les modules œdométriques $\mathrm{E}_{\text {oed }}$ ont été déterminés pour un tassement $\mathrm{s}_{100}$ correspondant à $100 \%$ de consolidation primaire et non pas à la déformation totale mesurée à la fin de chaque palier de chargement comme le préconisent ces méthodes d'essai. En effet, la consolidation secondaire qui se développe au-delà de $t_{100}$ réduit les valeurs de $E_{\text {oed }}$ sans raison puisqu'elle n'intervient pas pendant la consolidation primaire. De plus, conformément aux méthodes d'essai LPC (Magnan et al., 1985), seuls sont pris en compte pour 

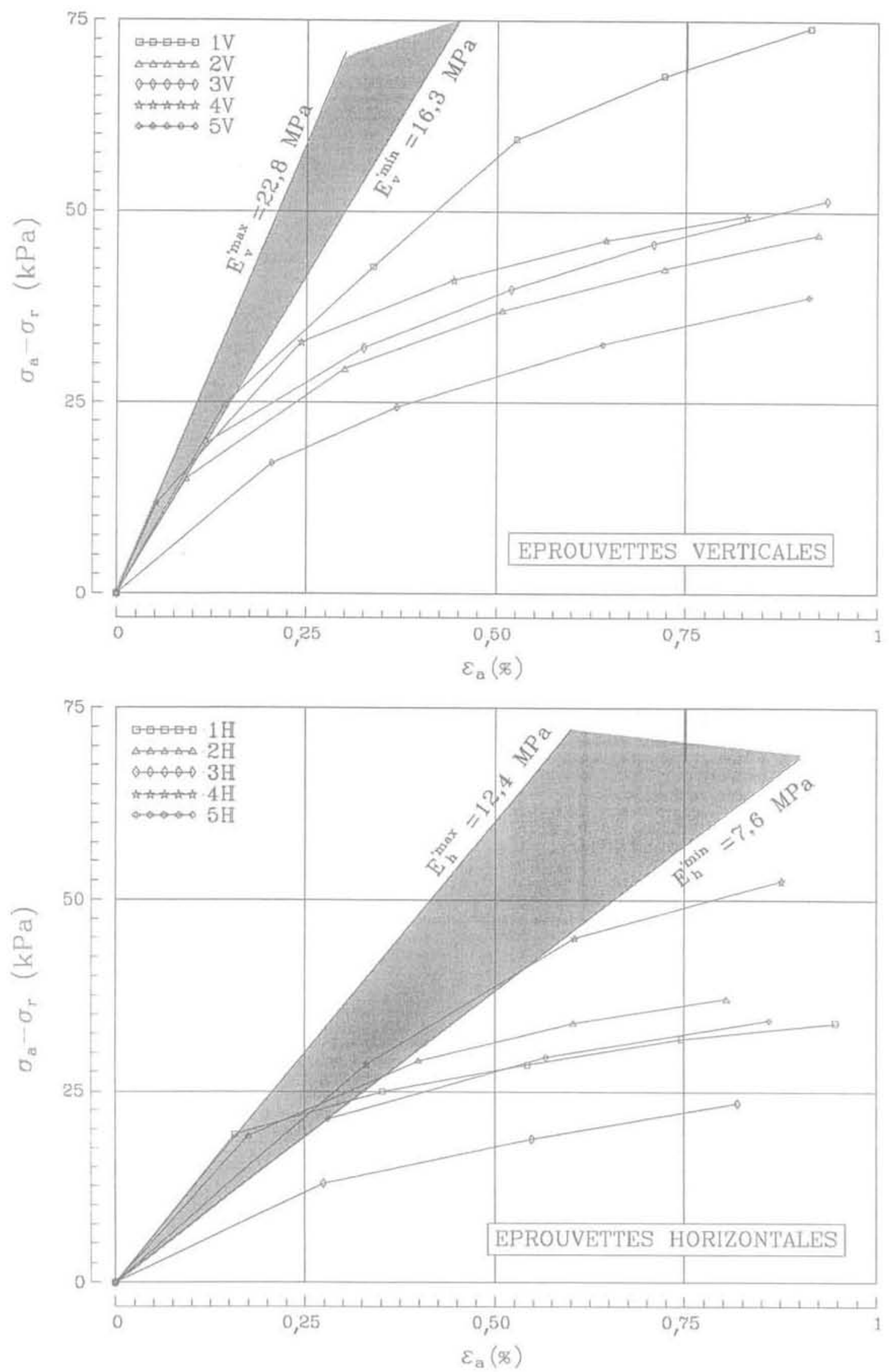

FIG. 17 Modules d'Young.

Young's modulus. 

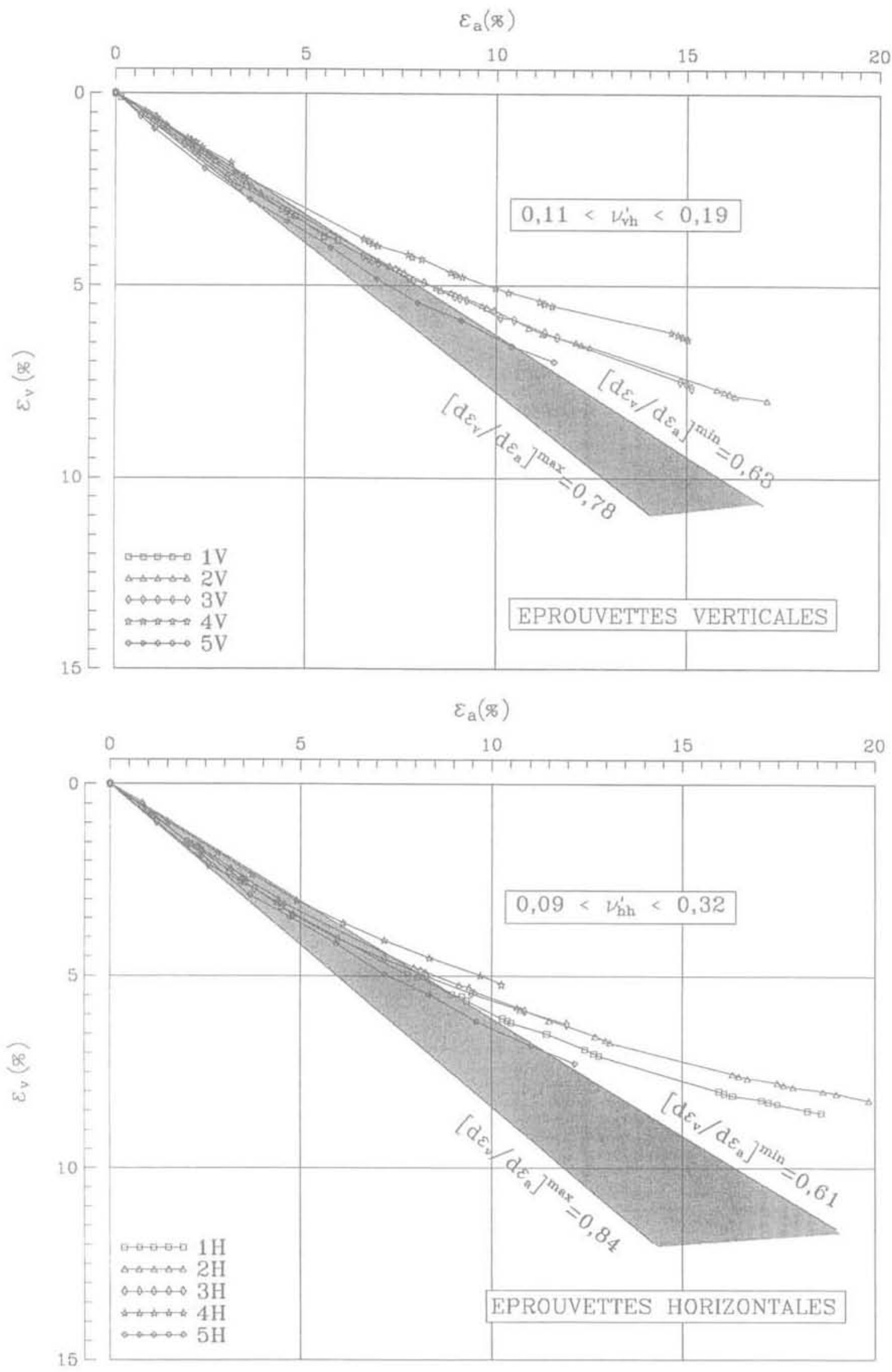

FIG. 18 Coefficients de Poisson.

Poisson's ratios. 

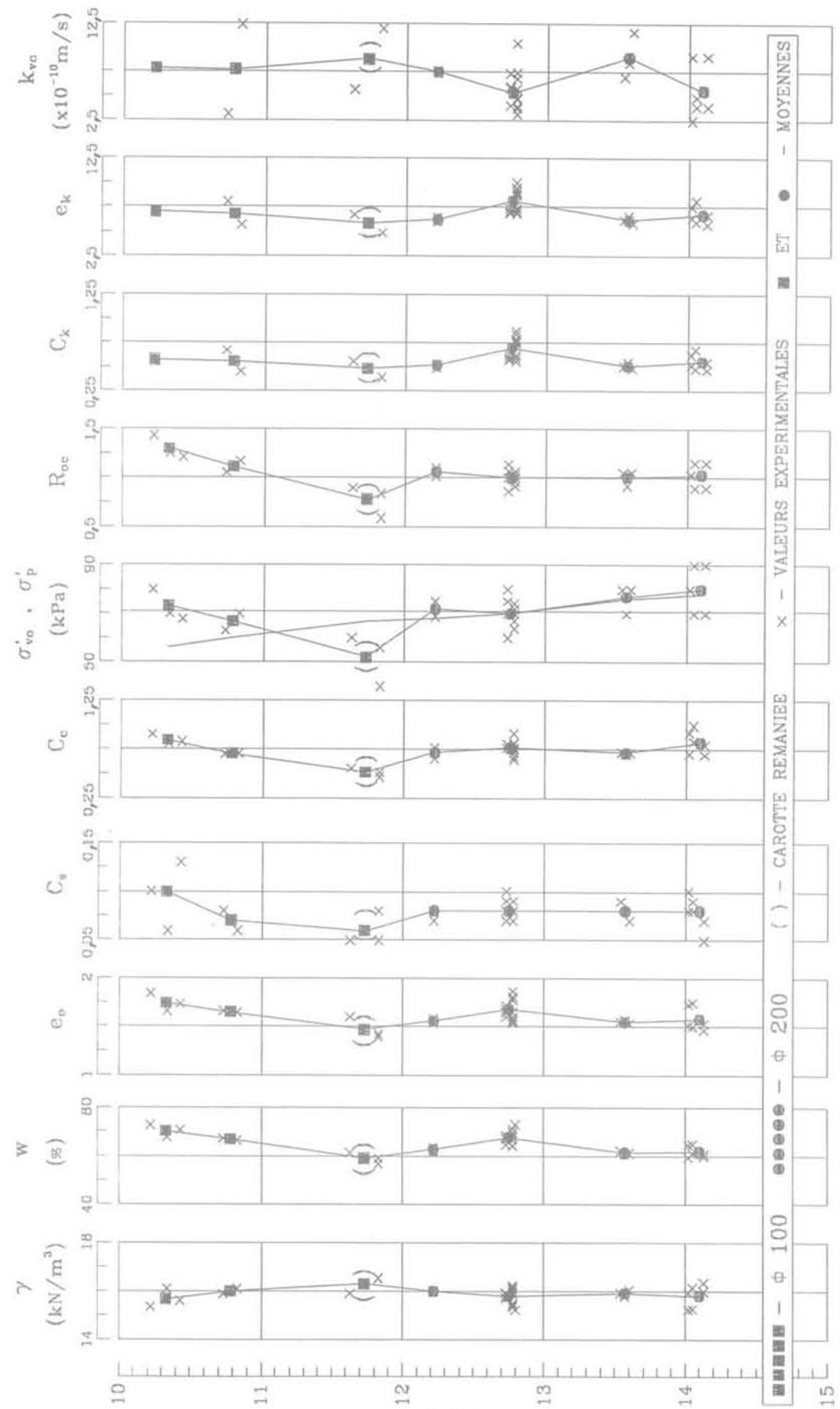

(ui) $\mathrm{z}$

FG. 19 Synthèse des résultats des essais cedométriques. Variation des paramètres physiques initiaux et des paramètres de compressibilité et de perméabilité en fonction de la profondeur.

Summary of oedometer test results. Variation of initial physical parameters, and compressibility and permeability parameters versus depth. 
caractériser la perméabilité les paliers de chargement au-delà de la pression de préconsolidation : la relation linéaire entre les indices des vides et le logarithme des coefficients de perméabilité est extrapolée ensuite vers l'indice des vides en place e, pour obtenir le coefficient de perméabilité verticale en place $k_{v}$. Le taux de variation de la perméabilité $C_{k}$ en fonction de l'indice des vides est la pente de cette relation linéaire.

Les résultats obtenus sur l'ensemble des essais œdométriques et rassemblés sur la figure 19 traduisent une assez bonne homogénéité des caractéristiques de perméabilité de l'argile de Guiche dans la couche étudiée, avec des valeurs de $k_{\text {comprises entre } 5,3.10^{-10}}$ et $9,1 \cdot 10^{-10} \mathrm{~m} / \mathrm{s}$ et de $C_{x}$ comprises entre 0,51 et 0,70 .

\section{5}

\section{Fluage}

Les courbes de fluage représentées sur la figure 4 ont des pentes comparables pour les charges dépassant la pression de préconsolidation lla courbe de fluage sous $420 \mathrm{kPa}$ présente une anomalie inexpliquée, peut-être due au remaniement de l'éprouvette). Les pentes des autres courbes sont environ trois fois plus faibles. Magnan (1992) a développé une analyse des courbes de fluage cedométrique dans laquelle le fluage sous de faibles charges tend progressivement vers la pente observée sous les fortes charges: l'accélération de la vitesse de tassement au-delà de 15000 ou 20000 min rend difficile le contrôle de cette hypothèse. Il n'est pas impossible, physiquement, que la vitesse de fluage du sol surconsolidé soit intrinsèquement plus faible que celle du sol dont la structure a été perturbée par le passage de l'état surconsolidé à l'état normalement consolidé.

Les résultats des huit essais de fluage ont été reportés sur le même diagramme de variation de la déformation verticale (respectivement de I'indice des vides) en fonction du logarithme de la contrainte effective, afin de construire un réseau de "lignes de temps " pour différentes durées de chargement (Fig. 20a) et la loi de fluage œdométrique associée (Fig. 20b). On observe un assez bon accord des données expérimentales avec ce modèle. La vitesse de fluage peut être décrite par le taux de fluage $C_{\alpha}$ ou l'indice de fluage $C_{\alpha t}$ $=\mathrm{C}_{i z}(1+\mathrm{e})$, selon la loi :

$$
\varepsilon_{v}=\varepsilon_{\mathrm{vi}}-\mathrm{C}_{\alpha} \lg \frac{\mathrm{t}}{\mathrm{t}_{\mathrm{i}}} \text { ou } \mathrm{e}=\mathrm{e}_{\mathrm{i}}-\mathrm{C}_{\mathrm{\alpha e}} \lg \frac{\mathrm{t}}{\mathrm{t}_{\mathrm{i}}}
$$

où e désigne l'indice des vides initial.

La pression de préconsolidation $\sigma^{\prime}$, dépend de la durée d'application de la charge selón la relation

$$
\frac{\sigma_{p}(t)}{\sigma_{p}\left(t_{i}\right)}=\left[\frac{t_{1}}{t}\right]^{C_{\alpha e} /\left(C_{c}-C_{p}\right)}
$$

D'autre part, la vitesse de déformation correspondant à la loi de fluage est égale à :

et la vitesse de variation de l'indice des vides est donnée par:

de sorte que la pression de préconsolidation est liée à la vitesse de déformation par la relation:

et à la vitesse de variation de l'indice des vides par la relation semblable :

Cette relation a été utilisée pour corriger les pres- sions de préconsolidation utilisées pour normaliser les résultats des essais triaxiaux en fonction de la vitesse de déformation axiale.

Les valeurs des paramètres de fluage déduites des courbes de la figure 20 sont les suivantes :

- indice de fluage : $\mathrm{C}_{\alpha r}=0,036$;

- pression de préconsolidation pour $t_{1}=1$ jour (1 $440 \mathrm{~min}$ ) : $\sigma_{\mathrm{p}}^{\prime}(\mathrm{t})=85 \mathrm{kPa}$;

- indice de compression : $\mathrm{C}_{\mathrm{c}}=0,78$;

- indice de gonflement : $\mathrm{C}_{\mathrm{s}}=0,07$;

- vitesse de référence : $6.10^{-8} \mathrm{~S}^{-1}$.

Les rapports $\mathrm{C}_{\mathrm{s}} / \mathrm{C}_{\mathrm{c}}=10$ et $\mathrm{C}_{\mathrm{ue}} / \mathrm{C}_{\mathrm{c}}=0,046$ sont typiques des sols compressibles et sont comparables, par exemple, aux valeurs trouvées pour les argiles de Mexico (Mesri et Choi, 1985) et de Cubzac-les-Ponts (Magnan et al., 1982).

\section{6}

\section{Conclusion}

Les essais triaxiaux et œdométriques réalisés sur l'argile molle faiblement organique et très plastique du site expérimental de Guiche, situé dans la basse vallée de l'Adour, ont permis de déterminer sur une épaisseur comprise entre dix et quinze mètres de profondeur: - une courbe d'état limite de forme sensiblement elliptique dans les plans $\left(s^{\prime}-t\right)$ et $\left(s^{\prime} / \sigma_{p}^{\prime}-t / \sigma_{p}^{\prime}\right)$, axée sur la droite caractérisant le rapport $\mathrm{K}_{0}$ des contraintes effectives verticale et horizontale des terres en place;

- une loi d'écoulement non associée dans ce même système de coordonnées;

- une anisotropie des résistances au cisaillement non drainé et une anisotropie de déformabilité caractérisée par des paramètres d'élasticité anisotrope ;

- une loi d'influence du temps sur le comportement de l'argile qui peut prendre la forme d'une loi de fluage ou d'une relation de la pression de préconsolidation avec la durée d'application des charges, ce qui permet de normaliser les courbes d'état limite correspondant à différentes vitesses de chargement par rapport à une vitesse de déformation de référence :

- une loi de compressibilité œdométrique classique typique des sols compressibles ;

- des relations linéaires entre le logarithme du coefficient de perméabilité et l'indice des vides.

Ces résultats sont comparables à ceux déjà obtenus antérieurement sur d'autres argiles molles faiblement organiques ou sensibles comme l'argile de Cubzac-lesPonts (Magnan et al., 1982), l'argile de Saint-Alban (Leroueil et al., 1979), l'argile de Londres (Graham et al., 1983), etc. Nous y voyons une preuve supplémentaire de la ressemblance des comportements des argiles molles dans toutes les régions du monde, quelles que soient leurs conditions de formation, la nature de leurs constituants minéraux et leur histoire géologique et hydrogéologique.

\section{Remerciements}

Les auteurs remercient MM. Jean-Paul Stempfelet et Marcel Daurade pour leur contribution à la réalisation du programme expérimental dont il est rendu compte dans cet article. 

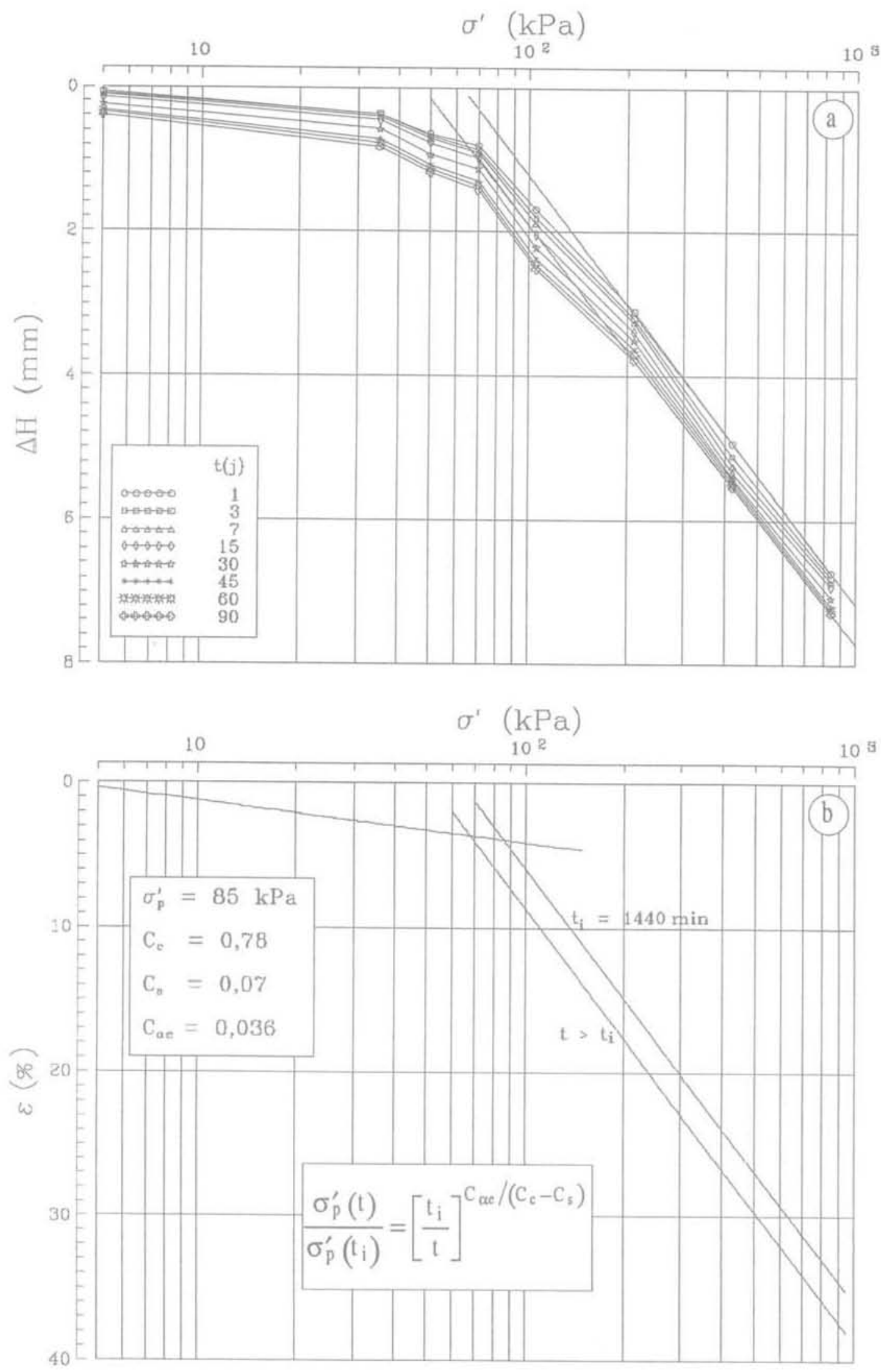

FG. 20 Fluage œdométrique de l'argile de Guiche (a/ réseau des « lignes de temps » pour différentes durées de chargement ; b/ loi de fluage œdométrique associée).

Oedometer creep of the Guiche clay (a/ set of « time lines w for various durations of loading : b/associated oedometer creep law). 
Atkinson J.H. - Anisotropic elastic deformation in laboratory undisturbed London clay. Geotechnique, 1975, vol. 25 (2). pp. $357-374$.

Bjerrum L. - Engineering geology of Norwegian normally consolidated marine clays as related to settlements of buildings. Geotechnique, 1967, vol. 17 (2), pp. 83-118.

Bjerrum L. - Problems of soil mechanics and construction on soft clays and structurally unstable soils. Proceedings of the 8th International Conference on Soll Mechanics and Foundation Engineering. Moscow, 1973, vol. 3, pp. 111-160

Félix B. - Le fluage et la consolidation unidimensionnelle des sols argileux. Rap. port de recherche LPC, 1980, n 94 , $174 \mathrm{p}$.

Josseaume H. Meimon Y.-Détermination de la loi de comportement des argiles naturelles. Bulletin de liaison des LPC 1976, tome 2, n spécial III, pp. 117-127.

Josseaume H., Blondeau F., Pilot G. Etude du comportement non drainé de trois argiles molles. Application au calcul des remblais, Bulletin de liaison des IPC, 1977, n ${ }^{\circ}$ spécial VI F, pp. 120-128

Josseaume H., Azizi F. - Détermination expérimentale de la courbe d'état limite d'une argile raide, l'argile verte du Sannoisien. Revue française de Géotechnique, 1991, n 54, pp. 13-25.

Josseaume H., Hieng O., Stempfelet J.-P. Détermination des paramètres de com pressibilité d'une argile raide à partit d'essais cedométriques à haute pression. Bulletin de liaison des LPC, 1991, $n^{\circ} 172$. pp. 109-120.

Graham J., Noonan M.L., Lew K.V. - Yield states and stress-strain relationship in natural plastic clay. Canadian Geotechnical Journal. 1983, vol. 20, pp. 502-516

Khemissa M. - Recherches experimentales sur les propriétés mécaniques d'une argile molle naturelle (argle de Guiche. vallée de l'Adour). Thèse de doctorat de
1'École nationale des ponts et chaussées, 1992,386 p.

Khemissa M., Magnan J.-P., Josseaume H. - Etude des propriétés mécaniques de largile molle de Guiche (vallée de l'Adour). Études et recherches des LPC. série Géotechnique, 1993, n GT 153 204 p.

Kenana A., Félix B. - Étude expérimentale du comportement visco-élastique d'une argile molle à l'appareil triaxial. Laboratoire central des ponts et chaussées. Paris, rapport des laboratoires, série Géotechnique, 1989, n GT 40, 99 p.

La Rochelle P. Sarrailh I. Tavenas F. Roy M. Leroueil S. - Causes of sampling disturbance and the design of a new sampler for sensitive soils. Canadian Geotechnical Journal, 1981, vol. 18 (1), pp. 52-66.

Lemasson $\mathrm{H}$. - Ensemble carottier à piston stationnaire-scissomètre. Bulletin de liaison des LPC, , 1973, n spécial T, pp. 276281

Leroueil S., Tavenas F., Le Bihan J.-P. Propriétés caractêristiques des arqiles de l'Est du Canada. Revue canadienne de Géotechnique, 1983. vol. 20 (4). pp. 681-705.

Leroueil S., Magnan J.-P.. Tavenas F. Remblais sur argiles molles. Technique et Documentation Lavoisier, Paris, 1985. $342 \mathrm{p}$.

Magnan J.-P. - Classification géotechnique des sols, A propos de la classification LPC. Bulletin de liaison des LPC, 1980 , $n^{\circ} 105$, pp. 49-52.

Magnan J.-P., Shahanguian S., Josseaume H. - Etude en laboratoire des états limites d'une argile molle organique. Revue francaise de Géotechnique, 1982. $n^{\circ} 33$, pp. 5-18

Magnan J.-P., Mieussens C., Soyez B., Vautrain J. - Essais cedométriques. Méthodes d'essai LPC, 1985, n-13, 83 p.

Magnan J.-P. Pival M. - Mesure des paramètres d'élasticité anisotrope de l'argile molle de Cubzac dans le domaine sur consolidé Revue francaise de Géotechnique, $1985, \mathrm{n}^{\circ} 33$, pp. 5-18.

Magnan J.-P., Bru J.-P. Pilot G. - Remblais d'essaj pour un tracé routier sur sol mou. Comptes rendus du $9^{e}$ Congrès europẻen de Mécanique des Sols et des Travaux de Fondations, Dublin. A.A. Balkema, Rotterdam, 1987, vol. 1. pp. 71-74.

Magnan J.-P. - Le rôle du fluage dans les calculs de consolidation et de tassement des sols compressibles. Bulletin de liaison des LPC, 1992, $\mathrm{n}^{\circ} 180$, pp. 19-24.

Meftah Kh. - Mécanique des sols anisotropes: caractérisation de l'anisotropie des sols et prise en compte dans le calcul des ouvrages. Thèse de doctorat de l'université Pierre-et-Marie-Curie, Paris VI, 1988, 284 p.

Mesri G. Choi Y.K. - Settlement analysis of embankments of soft clays. Proceedings of the 11th International Conference on Soil Mechanics and Foundation Engineering, San Francisco, 1985, vol. 2, pp. 441-464.

Piyal M., Magnan J.-P. - Paramètres d'élasticité anisotrope de l'argile molle organique de Cubzac-les-Ponts â l'état surconsolidé. Rapport de recherche L.PC. 1984, n 131, $108 \mathrm{p}$.

Queyroi D., Saint-Amand F. de, Magnan J.-P. - Les remblais d'essai de drains verticaux de l'autoroute A64 de Guiche (vallée de l'A dour). Bulletin de liaison des LPC, 1991, n¹76, pp. 57-72.

Tavenas F., Leroueil S. - Les concepts d'état limite et d'état critique et leurs applications pratiques à l'étude des argiles. Revue française de Géotechnique, $1979, \mathrm{n}^{\circ} 6$, pp. $27-49$.

Vogien M - Etude du comportement avant la rupture d'un remblai expérimental construit sur sols mous à Cubzac-lesPonts. Thèse de docteur-ingénieur, université Pierre-et-Marie-Curie, Paris VI, $1975,217 \mathrm{p}$ 